\title{
Molecular Mechanisms Controlling the Disease Cycle in the Vascular Pathogen Verticillium dahliae Characterized Through Forward Genetics and Transcriptomics
}

\author{
Jorge L. Sarmiento-Villamil, ${ }^{1,2}$ Nicolás E. García-Pedrajas, ${ }^{3}$ M. Carmen Cañizares, ${ }^{1}$ and \\ María D. García-Pedrajas ${ }^{1,+}$ \\ ${ }^{1}$ Instituto de Hortofruticultura Subtropical y Mediterránea "La Mayora"- Universidad de Málaga - Consejo Superior de \\ Investigaciones Científicas (IHSM-UMA-CSIC), Estación Experimental "La Mayora", 29750 Algarrobo-Costa, Málaga, Spain \\ ${ }^{2}$ Centre d'étude de la forêt (CEF) and Institut de biologie intégrative et des systèmes (IBIS), Université Laval, Québec QC G1V \\ OA6, Canada \\ ${ }^{3}$ Department of Computing and Numerical Analysis, C2 Building 3rd Floor, Campus Universitario de Rabanales, 14071 \\ Córdoba, Spain
}

Accepted 9 March 2020.

\begin{abstract}
The soil-borne pathogen Verticillium dahliae has a worldwide distribution and a plethora of hosts of agronomic value. Molecular analysis of virulence processes can identify targets for disease control. In this work, we compared the global gene transcription profile of random T-DNA insertion mutant strain D-10-8F, which exhibits reduced virulence and alterations in microsclerotium formation and polar growth, with that of the wild-type strain. Three genes identified as differentially expressed were selected for functional characterization. To produce deletion mutants, we developed an updated version of one-step construction of Agrobacterium-recombination-ready plasmids (OSCAR) that included the negative selection marker HSVtk (herpes simplex virus thymidine kinase gene) to prevent ectopic integration of the deletion constructs. Deletion of VdRGS1 (VDAG_00683), encoding a regulator of $G$ protein signaling (RGS) protein and highly upregulated in the wild type versus $D-10-8 F$, resulted in phenotypic alterations in development and virulence that were indistinguishable from those of the random T-DNA insertion mutant. In contrast, deletion of the other two genes selected, vrg1 (VDAG_07039) and vvs1 (VDAG_01858), showed that they do not play major roles in morphogenesis or virulence in $V$. dahliae. Taken together the results presented here on the transcriptomic analysis and phenotypic characterization of D-10-8F and $\triangle V$ VRGS1 strains provide evidence that variations in $G$ protein signaling control the progression of the disease cycle in $V$. dahliae. We propose that $G$ protein-mediated signals induce the expression of multiple virulence factors during biotrophic growth, whereas massive production of microsclerotia at late stages of
\end{abstract}

${ }^{\dagger}$ Corresponding author: M. D. García-Pedrajas; mariola@eelm.csic.es

Funding: This research was supported by the Spanish Ministry of Economy and Competitiveness grant AGL2016-80048-R, co-funded by the European Union (European Regional Development Fund).

*The $\boldsymbol{e}$-Xtra logo stands for "electronic extra" and indicates that four supplementary figures, one supplementary table, and one supplementary data file are published online.

The author(s) declare no conflict of interest.

(c) 2020 The American Phytopathological Society infection requires repression of $G$ protein signaling via upregulation of VdRGS1 activity.

Keywords: FlbA-like protein, gene deletion method, hemibiotrophic fungus, Verticillium wilt

Plant pathogens that invade their hosts systemically are frequently difficult to control, causing very destructive diseases. Verticillium dahliae is the main causal agent of Verticillium wilt, which affects a diversity of crops worldwide, posing a major problem to agriculture (Klosterman et al. 2011). Verticillium dahliae survives in the soil in the form of melanized resistant structures, the microsclerotia (MS), which are the main source of inoculum. In the presence of root exudates from host plants, the MS germinate, producing hyphae that penetrate the root cortex to reach the xylem. In the xylem vessels, both hyphal growth and conidiospores, which spread acropetally with the sap flow, contribute to vascular colonization (Yadeta and Thomma 2013). Verticillium dahliae can survive for long periods of time in the vascular system without causing obvious disease symptoms (Vallad and Subbarao 2008). Over time, clogging of water-conducting vessels by the fungus and by gums and tyloses released by the plant in response to infection, together with secretion of fungal toxins, results in disease symptoms that include wilting, stunting, and tissue chlorosis and necrosis (Santhanam et al. 2013; Yadeta and Thomma 2013; Zhou et al. 2012). In decaying plant tissue, V. dahliae undergoes a saprophytic phase in which leaf tissue is also colonized and large amounts of MS are produced. The MS are released to the soil, with the death and disintegration of plant tissue, where they can remain viable for long periods of time in the absence of host plants. Conidiospores are also produced in decaying plant tissue and may have a role in the dispersion of the fungus (Klosterman et al. 2009).

Successful infection and completion of the disease cycle is associated with the regulation of developmental processes and production of virulence factors and metabolic adaptation to the requirements of the different growth phases. Analysis of these processes at the molecular level can identify targets for disease control. As in other plant pathogens, forward and reverse genetic approaches have been applied to the identification of genes encoding virulence factors as well as regulators of 
morphogenesis in $V$. dahliae. In reverse genetics approaches, candidate genes to be studied are selected by homology with known genes, expression patterns, and genome comparisons. In recent years, the availability of the entire genome sequence of $V$. dahliae and other Verticillium species (Klosterman et al. 2011) has generated hundreds of candidate genes with potential relevant functions. Furthermore, the availability of the entire genome sequence facilitates the application of genome-wide transcription profiling and the identification of sets of genes that are induced or repressed in the conditions of interest. As a result, our knowledge of the regulatory pathways and cellular processes associated with key aspects of the life cycle of $V$. dahliae is rapidly expanding (Klimes et al. 2015).

Forward genetic approaches have long been used to gain insight into the molecular determinants of development and virulence in plant-pathogenic fungi. In these approaches, collections of random mutants are produced and those exhibiting phenotypic alterations of interest are genetically characterized. Even in the era of genomics, forward genetics approaches are of great interest, as they focus on mutants with clear phenotypic alterations and have great potential in the identification of novel regulators of virulence and morphogenesis in fungal pathogens. The method currently most widely used to generate collections of random mutants is insertional mutagenesis induced by Agrobacterium tumefaciens-mediated transformation (ATMT) (Mullins and Kang 2001; Santhanam 2012). T-DNA insertional
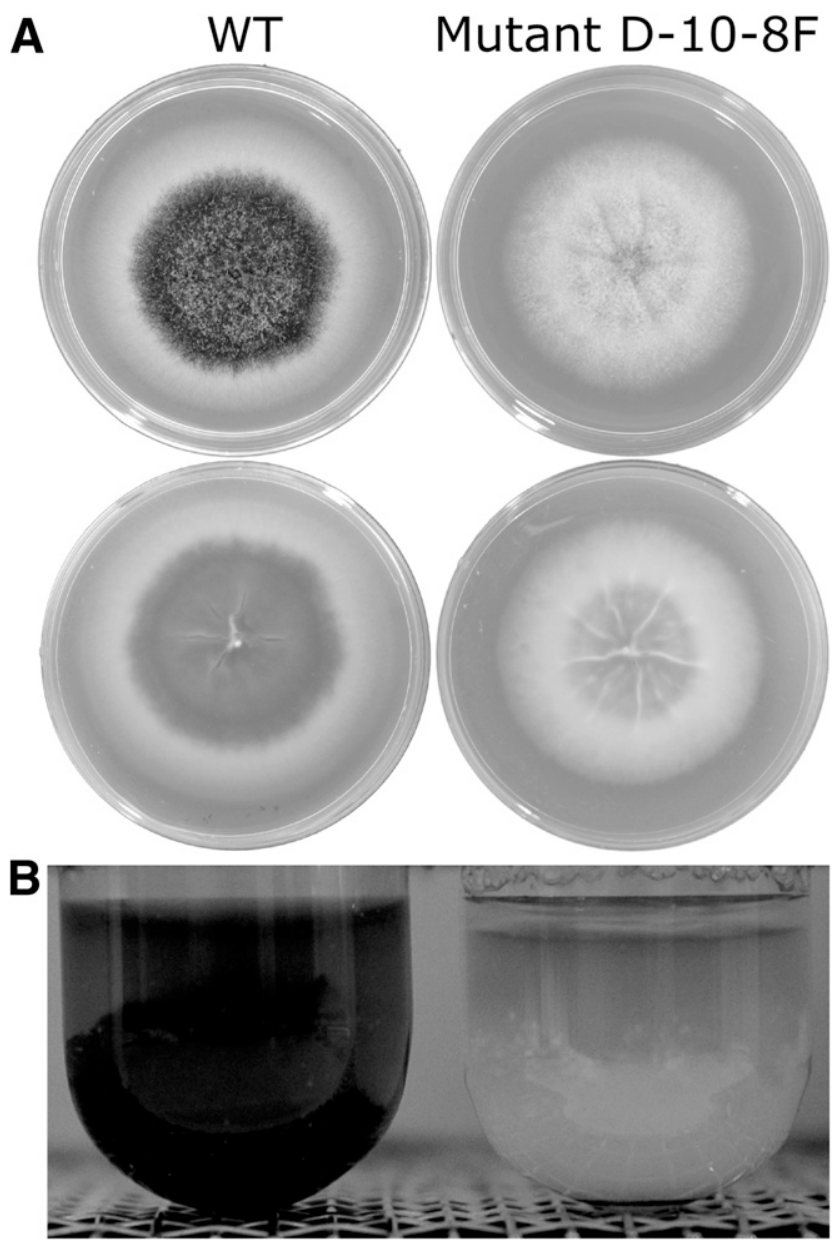

Fig. 1. Random T-DNA insertion mutant strain D-10-8F of Verticillium dahliae exhibits severely reduced microsclerotium formation. A, Colony morphology of the wild-type (WT) and random T-DNA insertion mutant D$10-8 \mathrm{~F}$ grown for 12 days on potato dextrose agar at $24^{\circ} \mathrm{C}$. B, WT and random mutant D-10-8F grown in Czapek Dox broth for 7 days at $24^{\circ} \mathrm{C}$ and $200 \mathrm{rpm}$. mutagenesis is a widely used tool in the identification of genes with important roles in virulence and development in $V$. dahliae (Gao et al. 2010; He et al. 2015; Luo et al. 2016; Maruthachalam et al. 2011; Santhanam et al. 2017; SarmientoVillamil et al. 2018; Xie et al. 2017; Zhang et al. 2015a, 2018, 2019).

Whereas different approaches can be used to identify genes with potential roles of interest, targeted deletion is the key technique for their functional analysis. To speed this process in the era of genomics, efforts have been made to simplify both the steps required to generate deletion constructs and the identification of deletion mutants upon fungal transformation. In $V$. dahliae, ATMT is the preferred fungal transformation method since its initial successful application to the disruption of a trypsin protease gene (Dobinson et al. 2004). The approaches commonly used for targeted gene deletion in this fungus involved, therefore, the generation of deletion constructs in binary vectors compatible with ATMT (Paz et al. 2011; Wang et al. 2016). Recently, a modification was introduced to the methodology developed by Paz et al. (2011) so that, without increasing the number of steps involved, deletion mutants are routinely green fluorescent protein (GFP)-tagged and can be characterized through fluorescent microscopic techniques (Sarmiento-Villamil et al. 2018).

The availability of the entire genome sequence of $V$. dahliae allows exploiting random mutants with phenotypes of interest beyond the identification of individual genes affected by $\mathrm{T}$ DNA insertions. In this work, we selected random T-DNA insertion mutant D-10-8F, exhibiting a significant reduction in virulence and altered MS development and polar growth, and characterized it by comparing its global gene transcription pattern with that of the parental strain. Several of the genes identified as differentially expressed were functionally characterized by production of deletion mutants. For this purpose, we used the OSCAR method (Gold et al. 2017; Paz et al. 2011) but introduced a negative selection marker in the pOSCAR vector to prevent random integration of the transforming construct, thus greatly facilitating the selection of deletion mutants. The results presented here have led us to propose a model on the role played by activation/inactivation of $\mathrm{G}$ protein signaling in the progression of the $V$. dahliae disease cycle.

\section{RESULTS}

\section{Identification of a random T-DNA insertion mutant in $V$. dahliae exhibiting important morphological alterations and reduced virulence.}

When using OSCAR constructs, which are generated in binary vectors compatible with ATMT (Gold et al. 2017; Paz et al. 2011), to produce deletion mutants in V. dahliae, those transformants that do not exhibit deletion of the target gene but ectopic integration of the T-DNA were not discarded but were collected and screened for the presence of visible phenotypic alterations in in-vitro culture (Sarmiento-Villamil et al. 2018). Random T-DNA insertion mutant D-10-8F exhibited clear morphological alterations. On potato dextrose agar (PDA), colonies of D-10-8F showed a marked reduction in the production of MS (Fig. 1A). Growth in Czapex dox broth further established the strong defect in MS production exhibited by the random mutant. In this liquid medium, the wild-type (WT) strain Dvd-T5 produced large amounts of MS that resulted in highly pigmented cultures, whereas cultures of mutant D-10-8F remained unpigmented and appeared to contain no MS (Fig. 1B). We also assessed growth of WT and random mutant strains on PDA covered with cellophane membranes (Fig. 2A). Microscopic observations showed that, as previously reported (Sarmiento-Villamil et al. 2018), in this growth condition, the 
parental WT strain Dvd-T5 formed numerous branches with swollen tips that resemble hyphopodia (Fig. 2B, left panels). In contrast, D-10-8F displayed hyperpolarized growth on cellophane, producing bundles of hyphae that exhibited very little branching (Fig. 2B, right panels).

Pathogenicity assays, using Nicotiana benthamiana as a host, showed that random T-DNA insertion mutant D-10-8F was also impaired in its ability to induce Verticillium wilt symptoms (Table 1). When symptoms in plants inoculated with D-10-8F and WT were compared, there were significant differences among treatments and time after inoculation and their interaction $(P<0.0001)$ indicating a significant increase in disease severity in at least one of the treatments (analysis of variance $\mathrm{F}$ [ANOVA-F]). Initially, at 4 weeks postinoculation (wpi), the mutant $\mathrm{D}-10-8 \mathrm{~F}$ caused as much disease as the $V$. dahliae parental WT strain $(P=0.1308)$. However, in D-10$8 \mathrm{~F}$ treatments, disease symptoms did not progress thereafter, whereas in WT infections there was a significant increase in disease severity values over time (Table 1).

\section{Microarray analysis reveals major differences in global gene expression between the WT and the random T-DNA insertion mutant.}

In order to further characterize mutant strain D-10-8F, we performed a microarray analysis comparing its global transcription pattern with that of the WT parental strain Dvd-T5. For that, RNAs were extracted from both strains grown for $48 \mathrm{~h}$ on PDA covered with cellophane. We hypothesized that the growth condition selected would allow us to identify genes differentially expressed in the two strains involved in both developmental processes, such as MS formation and polarized growth and virulence. The microarray analysis showed that the clear phenotypic differences of WT and D-10-8F correlated with considerable differences in global gene transcription patterns. Thus, 566 genes with differential expression ratios of at least twofold were detected, 206 of them were up-regulated in the WT and 360 were down-regulated in the WT, as compared with their expression in random mutant D-10-8F. To validate microarray data, reverse transcription quantitative real time PCR (RT-qPCR) was conducted to confirm differential expression in seven selected genes. Gene expression profiles determined by both microarray and RT-qPCR show the same trend in all seven genes, with some variation in the magnitude of differential expression (Table 2).

The 566 differentially expressed genes were assigned to different biological and molecular functional categories according to the Gene Ontology (GO) Consortium database. A number of functional categories were clearly enriched with genes that exhibited higher levels of expression in random mutant D-10-8F than in WT, for instance, the molecular function categories of oxidoreductases, hydrolases, and lyases (Fig. 3A). Of the downregulated genes in WT, $14 \%$ potentially had oxidoreductase activity, whereas only $5 \%$ of the genes identified as upregulated in the WT fell in that category (Fig. 3A). Similar differences were observed in genes potentially displaying hydrolase activity; 12 and $6 \%$ of the genes identified as down- and upregulated in the WT, respectively, were classified as hydrolases. The classification in biological process categories identified genes encoding proteins involved in metabolism as the larger group; roughly 24 and $18 \%$ of the genes identified as repressed and induced, respectively, in WT versus the random mutant (Fig. 3B). Oxidation-reduction biological processes were clearly induced in the mutant; $19 \%$ of the genes that were upregulated in the mutant versus WT fell in this category. The GO analysis also showed that $5 \%$ of the genes that were upregulated in the mutant versus WT were potentially involved in proteolysis, whereas no genes from this category were identified as upregulated in the WT (Fig. 3B). The percentage of genes that could not be assigned to any biological process or molecular function category was significantly higher in the population of genes that were up-regulated in WT than in those that were down-regulated in this strain (Fig. 3A and B).

The potential subcellular localization of the proteins encoded by the 566 differentially expressed genes was also determined
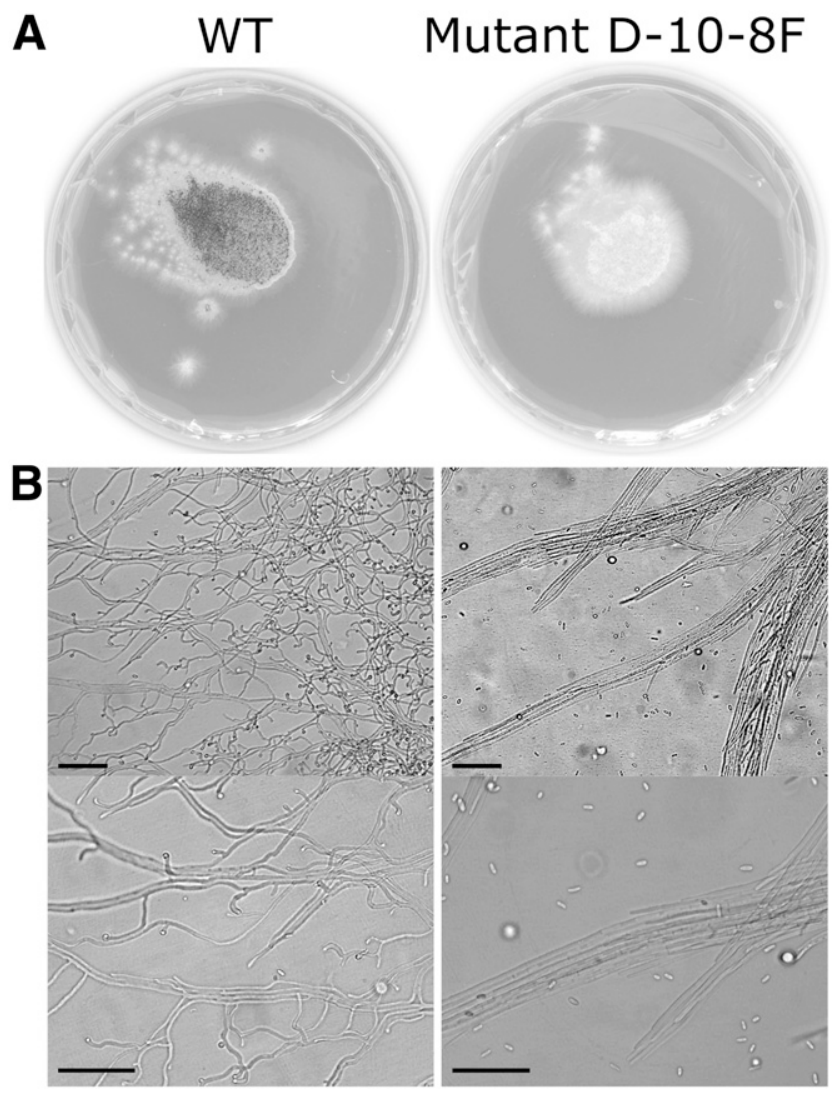

Fig. 2. Random T-DNA insertion mutant strain D-10-8F of Verticillium dahliae exhibits hyperpolarized growth. A, Wild type (WT) and random mutant grown for 4 days on potato dextrose agar (PDA) medium covered with cellophane membranes. Plates were inoculated with $10^{5}$ conidia of each strain and were incubated at $24^{\circ} \mathrm{C}$ in the dark. B, Microscopic observation of strains grown on PDA covered with cellophane for 3 days. Scale bar $=50 \mu \mathrm{m}$.

Table 1. Disease severity of Nicotiana benthamiana plants inoculated with Verticillium dahliae wild type and random T-DNA insertion mutant D-10-8F

\begin{tabular}{lcccc}
\hline & & \multicolumn{3}{c}{ Disease severity } \\
\cline { 3 - 5 } Treatment & & & \multicolumn{2}{c}{$\mathbf{9 5 \%}$ confidence interval } \\
\cline { 3 - 5 } Time & & RME $^{\mathbf{b}}$ & Lower bound & Upper bound \\
\hline Mock & 4 & 0.27819 & 0.2436 & 0.31641 \\
& 5 & 0.27819 & 0.2436 & 0.31641 \\
& 6 & 0.27819 & 0.2436 & 0.31641 \\
Wild type & 7 & 0.27819 & 0.2436 & 0.31641 \\
& 4 & 0.56693 & 0.51463 & 0.61752 \\
& 5 & 0.67618 & 0.6125 & 0.73277 \\
& 6 & 0.72992 & 0.64599 & 0.79731 \\
D-10-8F & 7 & 0.76254 & 0.66167 & 0.83589 \\
& 4 & 0.49236 & 0.44378 & 0.54111 \\
& 5 & 0.52765 & 0.47116 & 0.5833 \\
& 6 & 0.55976 & 0.49709 & 0.62023 \\
& 7 & 0.57191 & 0.50485 & 0.63595 \\
\hline
\end{tabular}

\footnotetext{
Number of weeks postinoculation.

${ }^{\mathrm{b}} \mathrm{RME}=$ Relative marginal effects.
} 
(Supplementary Data File S1). Close to $8 \%$ of V. dahliae genes are predicted to encode secreted proteins (Klosterman et al. 2011). Interestingly, $30 \%$ of the 360 genes whose expression was induced in the mutant versus WT and $23 \%$ of the 206 genes exhibiting higher levels of expression in the WT than in the mutant were predicted to encode secreted proteins. Other categories of subcellular location showed noticeable differences in the proteins encoded by genes that were up- and down-regulated

Table 2. Reverse transcription quantitative real time PCR (RT-qPCR) validation of microarray data in selected Verticillium dahliae genes

\begin{tabular}{|c|c|c|c|c|c|c|}
\hline \multirow[b]{3}{*}{ Gene ID } & \multicolumn{3}{|c|}{ Mean fold changes in wild type vs. mutant } & \multicolumn{3}{|c|}{$P$ value } \\
\hline & \multicolumn{2}{|c|}{ RT-qPCR } & \multirow[b]{2}{*}{ Microarray } & \multicolumn{2}{|c|}{ RT-qPCR } & \multirow[b]{2}{*}{ Microarray } \\
\hline & $\beta$-actine ${ }^{a}$ & GAPDH $^{\mathbf{a}, \mathbf{b}}$ & & $\beta$-actine ${ }^{a}$ & GAPDH $^{\mathbf{a}, \mathbf{b}}$ & \\
\hline VDAG_07904 & -2.15 & -3.34 & -2.17 & $<0.001$ & $<0.001$ & $<0.001$ \\
\hline VDAG_08233 & -0.93 & -2.12 & -1.96 & 0.05 & $<0.001$ & $<0.001$ \\
\hline$V D A G \_04977$ & 4.86 & 3.67 & 2.74 & $<0.001$ & $<0.001$ & $<0.001$ \\
\hline$V D A G \_02980$ & 1.55 & 0.94 & 1.41 & 0.02 & 0.02 & 0.02 \\
\hline VDAG_06199 & 5.72 & 4.53 & 1.77 & $<0.001$ & $<0.001$ & 0.007 \\
\hline$V D A G \_07183$ & 5.05 & 3.86 & 2.30 & $<0.001$ & $<0.001$ & $<0.001$ \\
\hline$V D A G \_10193$ & 2.53 & 1.34 & 1.09 & $<0.001$ & 0.02 & $<0.001$ \\
\hline
\end{tabular}

${ }^{a}$ Housekeeping genes used to assess the expression level of each gene in the different samples.

b Glyceraldehyde 3-phosphate dehydrogenase.

\section{A Molecular Function}

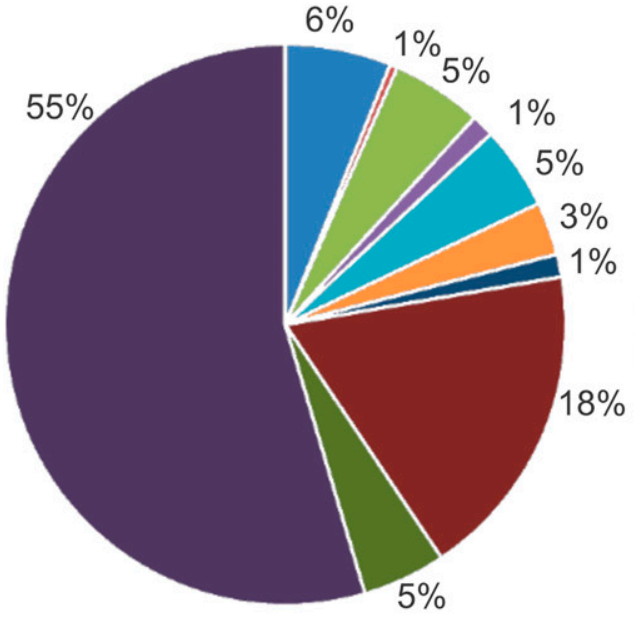

Up-regulated

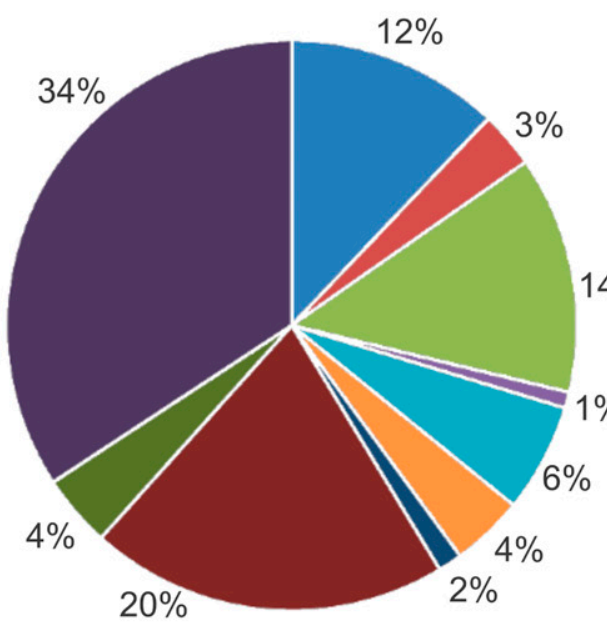

Down-regulated
- Hydrolase activity

- Lyase activity

- Oxidoreductase activity

- Ligase activity

$14 \%$ "Transporter activity

- Transferase activity

- Transcription factor activity

- Binding

- Others

- No GO terms identified

B

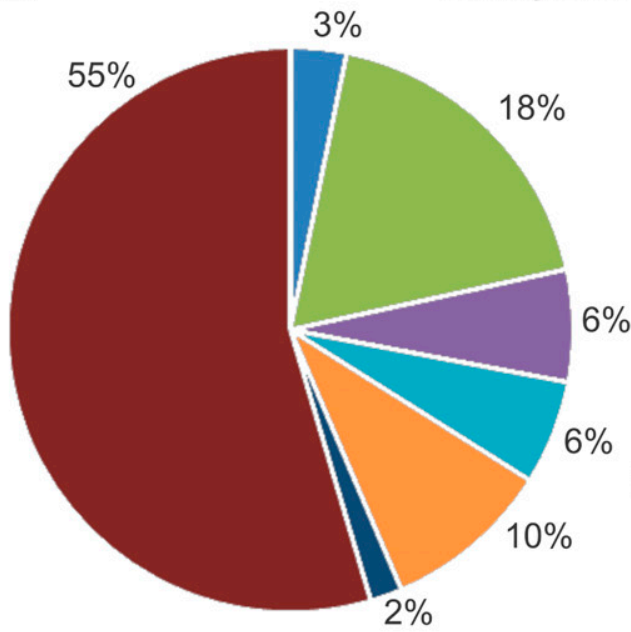

Up-regulated

Biological Process

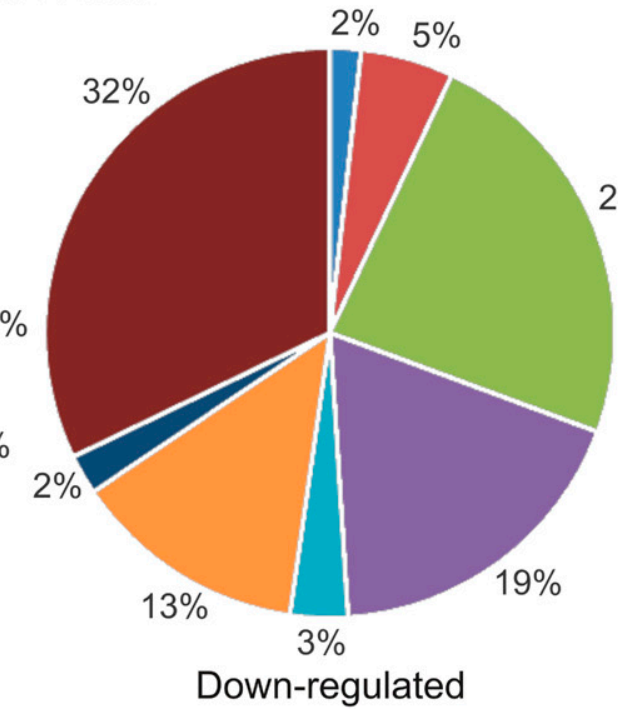

- Development
$24 \%$ - Proteolysis
" Metabolic process

- Oxidation-reduction process

- Biological regulation

- Transport

- Others

- No GO terms identified

Fig. 3. Functional categories of the Verticillium dahliae genes differentially expressed in wild type (WT) versus random T-DNA insertion mutant D-10-8F. A, The 206 genes with higher levels of expression in the WT than in the mutant (left) and the 360 genes with lower levels of expression in the WT than in the mutant (right) were assigned to several molecular function and B, biological process categories according to the Gene Ontology Consortium database. 
in WT versus mutant. For instance, 5.3 and $16.5 \%$ of the genes that were up- and down-regulated in the mutant versus WT, respectively, were predicted to encode proteins localized to the nucleus. The percentage of genes encoding mitochondrial proteins was, on the other hand, more than double in the population of those that were up-regulated in $\mathrm{D}-10-8 \mathrm{~F}$ than in the ones more highly expressed in WT (7.8 vs. 3.4\%). Similarly, 11.7 and $4.4 \%$ of the genes that were up- and down-regulated in the mutant versus WT, respectively, were predicted to encode peroxisomal proteins.

\section{Expression patterns of genes associated with oxidation- reduction processes and primary and secondary metabolism are significantly altered in the random mutant.}

A large percentage of genes exhibiting higher levels of expression in D-10-8F than in the WT encoded proteins potentially associated with oxidation-reduction processes (Fig. 3). Among the oxidoreductase genes whose expression was highly up-regulated in D-10-8F versus WT, there were two encoding succinatesemialdehyde dehydrogenases (SSADHs), VDAG_03345 and $V D A G \_04572$ (Table 3). SSADHs are involved in $\gamma$-aminobutyric acid (GABA) catabolism. VDAG_04571, adjacent to one of the SSADH genes and also highly up-regulated in D-10-8F $\left(\log _{2}\right.$ fold change $[\mathrm{FC}]=4.69$ ), encoded another enzyme involved in GABA catabolism, 4-aminobutyrate aminotransferase. A second 4-aminobutyrate aminotransferase-encoding gene, VDAG_06397, also exhibited higher levels of expression in the random mutant than in the WT. This GABA degradation produces succinate and is a biochemical bypass of the tricarboxylic acid cycle called the GABA shunt. GABA can be produced from glutamate through the action of a glutamate decarboxylase but also by the oxidative deamination of polyamines (PA), which requires amine oxidases. These include copper-containing amine oxidases (CuAOs) and flavin-containing PA oxidases (PAOs) (Angelini et al. 2010). Interestingly, a cluster of three genes putatively encoding a $\mathrm{CuAO}$ (VDAG_06374), a transporter protein similar to GABA permeases (VDAG_06373), and an aldehyde dehydrogenase (VDAG_06372) was highly up-regulated in the mutant versus WT. This cluster could be involved in the production of GABA from the PA putrescine, which requires both deamine oxidase and aldehyde dehydrogenase activities. Furthermore, VDAG_02801 encoding a protein highly similar to PAOs exhibited a 3.4-fold increased expression in D-10-8F versus WT (Table 3).

The reaction products of the GABA shunt enzymes identified as upregulated in the random mutant include redox equivalents that protect the cell from damage caused by reactive oxygen species (Bönnighausen et al. 2015). Several other genes potentially involved in oxidative stress responses were identified as differentially expressed. These included three catalase genes, $V D A G \_05792, V D A G \_09115$, and VDAG_04826, and superoxide dismutase genes $V D A G \_07230$ and $V D A G \_08724$; all of them except $V D A G \_08724$ were up-regulated in D-10-8F. A quinone oxidoreductase gene, $V D A G \_07223$, was expressed at fivefold higher levels in the mutant strain than in the WT. Quinone oxidoreductases have a role as superoxide scavengers (Siegel et al. 2004). Five genes encoding PA transporters highly similar to Saccharomyces cerevisiae Tpo1 and other proteins of the same family of transporters were up-regulated in D$10-8 \mathrm{~F}$ versus WT. CuAOs and PAOs are major sources of $\mathrm{H}_{2} \mathrm{O}_{2}$ via the catabolism of PAs, and Tpo1 plays a role in the control of the cell cycle and the expression of antioxidant proteins during oxidative stress by mediating the export of PAs (Krüger et al. 2013). Several genes encoding proteins with a potential role in nitrosative stress responses were identified as differentially expressed. A key nitric oxide (NO) detoxification mechanism involves flavohemoglobins
(Turrión-Gómez et al. 2010); VDAG_10220, one of the two $V$. dahliae genes potentially encoding flavohemoglobins, was twofold up-regulated in the random mutant versus WT. Two $S$ (hydroxymethyl)-glutathione dehydrogenase genes, VDAG_06717 and VDAG_00207, were equally up-regulated in D-10-8F. The latter encodes a protein highly similar to MoSfa1, involved in NO detoxification in Magnaporthe oryzae and required for full virulence (Zhang et al. 2015b).

Carbohydrate active enzyme (CAZy) genes were identified as both up- and downregulated in WT versus mutant. $V D A G \_03236$, the CAZy gene with the highest level of induction in WT as compared with its expression in the mutant (Table 3 ), encodes a putative $\alpha$-galactosidase very similar to Mel1 and other closely related melibiases from $S$. cerevisiae, which break down melibiose into galactose and glucose (Gasent-Ramírez et al. 1995). The adjacent gene VDAG_03237, also highly upregulated in WT, with a $\log _{2} \mathrm{FC}$ value of 4.39 , encodes a putative maltose permease (Table 3 ). Other genes encoding $\alpha$-galactosidases similar to melibiases were upregulated in WT (Table 3). VDAG_03792, encoding a putative invertase ( $\beta$-fructofuranosidase) was moderately up-regulated in WT versus mutant strain D-10-8F. Invertases produce melibiose from the trisaccharide raffinose by releasing the fructose moiety. The VDAG_03236 and VDAG_03792 proteins were both predicted to be extracellular. The $\alpha$-glucosidase gene $V D A G \_02814$, the glucoamylase P gene VDAG_00408, and two putative $\beta$-glucosidase genes, VDAG_09750 and VDGA_01263, were all upregulated in the WT, as compared with their expression in the mutant, and were predicted to encode extracellular proteins. Glucoamylases and $\alpha$-glucosidases are amylolytic enzymes involved in starch degradation, whereas $\beta$-glucosidases act on cellulose. Many of the CAZy genes showing the opposite expression pattern, that is higher expression in the random mutant than in WT, encoded putatively secreted proteins involved in the degradation of plant cell walls. Eleven genes potentially encoding pectinolytic enzymes that are also part of the $V$. dahliae secretome were identified as differentially expressed and, interestingly, all of them were up-regulated in the mutant strain D-10-8F (Table 3).

Several pathways of secondary metabolism appeared to be up-regulated in mutant strain D-10-8F (Table 4). One of the gene clusters that were significantly up-regulated in the mutant encodes a small cysteine-rich protein with a LysM domain and a predicted signal peptide (VDAG_00902). Zhou et al. (2017) found that transcription of this gene, which they designated $V d S C P 9$, is induced when $V$. dahliae is grown on cellophane or on root surfaces. A second cluster that was up-regulated in D-10$8 \mathrm{~F}$ is composed of four genes and one of them encodes a TRI14like protein. In Fusarium graminearum, TRI14 is required for increased virulence and deoxynivalenol (DON) production on wheat (Dyer et al. 2005). VDAG_09534, encoding a polyketide synthase (PKS), and a number of genes that are potentially part of the same gene cluster were also highly induced in the mutant (Table 4). A few genes potentially associated with secondary metabolism were identified as upregulated in the WT, for example the PKS gene VDAG_08448. However, no other genes of the putative cluster containing VDAG_08448 showed differential expression in WT versus mutant. $V D A G \_05055$ and $V D A G \_10292$, two genes encoding proteins harboring the DUF3328 motif associated with the synthesis of ustiloxin (Ye et al. 2016), were both up-regulated in the WT.

\section{The transcription profile of the random mutant shows upregulation of different activities potentially associated with pathogenesis.}

Numerous genes with predicted functions in pathogenesis were identified as upregulated in the mutant strain in addition to those encoding CAZy and pectinolytic enzymes mentioned 
Table 3. Selected Verticillium dahliae genes identified as differentially expressed in wild type versus random T-DNA insertion mutant D-10-8F

\begin{tabular}{|c|c|c|}
\hline Gene ID & WT vs. mutant ${ }^{a}$ & Functional annotation \\
\hline \multicolumn{3}{|c|}{ Genes encoding CAZys and pectinolytic enzymes } \\
\hline$V D A G \_03236$ & 3.98 & $\alpha$-Galactosidase \\
\hline$V D A G \_01555$ & 2.99 & $\alpha$-Glucosidase \\
\hline$V D A G \_02814$ & 1.96 & Glucan $1,3-\beta$-glucosidase \\
\hline$V D A G \_00408$ & 1.92 & Glucoamylase P \\
\hline$V D A G \_09750$ & 1.55 & $\beta$-Glucosidase \\
\hline$V D A G \_08038$ & 1.38 & Endo $\alpha-1,4$ polygalactosaminidase \\
\hline$V D A G \_01263$ & 1.17 & $\beta$-Glucosidase \\
\hline$V D A G \_02274$ & 1.17 & $\alpha$-Galactosidase \\
\hline$V D A G \_03792$ & 1.02 & $\beta$-fructofuranosidase (invertase) \\
\hline VDAG_02907 & 1.02 & $\alpha$-Galactosidase \\
\hline$V D A G \_07881$ & -3.82 & Pectinesterase \\
\hline$V D A G \_04977$ & -2.74 & Endopolygalacturonase \\
\hline$V D A G \_06254$ & -2.64 & Endoglucanase-5 \\
\hline$V D A G \_09727$ & -2.52 & Cellulose growth-specific protein \\
\hline$V D A G \_02959$ & -2.47 & Endoglucanase-4 \\
\hline$V D A G \_07238$ & -2.41 & Pectin lyase \\
\hline$V D A G \_08741$ & -2.40 & Endochitinase \\
\hline VDAG_06155 & -2.28 & Pectate lyase \\
\hline$V D A G \_09523$ & -2.12 & Pectate lyase D \\
\hline VDAG_07144 & -1.84 & Pectin lyase regulated by Snif1 \\
\hline$V D A G \_03707$ & -1.60 & $\alpha-N$-arabinofuranosidase \\
\hline$V D A G \_03708$ & -1.51 & Arabinan endo-1,5- $\alpha$-L-arabinosidase A \\
\hline$V D A G \_06165$ & -1.44 & Endo- $1,4-\beta$-xylanase A regulated by Snif1 \\
\hline$V D A G \_09063$ & -1.40 & Rhamnogalacturonase B \\
\hline$V D A G \_04336$ & -1.38 & $\alpha-N$-arabinofuranosidase \\
\hline$V D A G \_02231$ & -1.34 & Feruloyl esterase B \\
\hline VDAG_05344 & -1.33 & Pectate lyase B regulated by Snif1 \\
\hline$V D A G \_00063$ & -1.16 & Pectate lyase \\
\hline VDAG_08954 & -1.16 & Feruloyl esterase B \\
\hline$V D A G \_10193$ & -1.09 & Cellulose-binding family II \\
\hline
\end{tabular}

Genes related to oxidation-reduction processes and oxidative and nitrosative stress

\begin{tabular}{|c|c|c|}
\hline$V D A G \_08724$ & 1.28 & Cytosolic $\mathrm{Cu} / \mathrm{Zn}$ superoxide dismutase \\
\hline$V D A G \_03345$ & -5.03 & Succinate-semialdehyde dehydrogenase \\
\hline$V D A G \_04572$ & -4.81 & Succinate-semialdehyde dehydrogenase \\
\hline$V D A G \_04571$ & -4.69 & 4-Aminobutyrate aminotransferase \\
\hline$V D A G \_06374$ & -2.58 & Peroxisomal copper amine oxidase \\
\hline$V D A G \_07223$ & -2.41 & Quinone oxidoreductase \\
\hline$V D A G \_07230$ & -2.29 & Superoxide dismutase \\
\hline$V D A G \_06372$ & -2.03 & Aldehyde dehydrogenase \\
\hline$V D A G \_05792$ & -2.01 & Peroxisomal catalase \\
\hline$V D A G \_06397$ & -1.82 & 4-Aminobutyrate aminotransferase \\
\hline$V D A G \_02801$ & -1.77 & Polyamine oxidase \\
\hline$V D A G \_06717$ & -1.75 & $\begin{array}{l}S \text {-(hydroxymethyl) glutathione } \\
\text { dehydrogenase }\end{array}$ \\
\hline$V D A G \_00207$ & -1.28 & $\begin{array}{l}S \text {-(hydroxymethyl) glutathione } \\
\text { dehydrogenase (MoSfa1) }\end{array}$ \\
\hline$V D A G \_09115$ & -1.23 & Catalase \\
\hline$V D A G \_10220$ & -1.22 & Flavohemoglobin \\
\hline$V D A G \_04826$ & -1.06 & Peroxidase/catalase \\
\hline
\end{tabular}

Genes potentially associated with pathogenesis and development

\begin{tabular}{|c|c|c|}
\hline$V D A G \_10456$ & 2.38 & Similar to $\beta$-Ig-H3/Fasciclin \\
\hline$V D A G \_07271$ & 1.90 & Tubulin-tyrosine ligase domain \\
\hline$V D A G \_03514$ & 1.64 & Similar to flocculation proteins \\
\hline$V D A G \_07929$ & 1.40 & Integral membrane protein, CFEM domain \\
\hline$V D A G \_07991$ & 1.35 & $\begin{array}{l}\text { Similar to biotrophy-associated secreted } \\
\text { proteins }\end{array}$ \\
\hline$V D A G \_05055$ & 1.15 & DUF3328 domain protein \\
\hline$V D A G_{-} 10292$ & 1.10 & DUF3328 domain protein \\
\hline$V D A G \_05405$ & -3.80 & Chitin synthase \\
\hline$V D A G \_00902$ & -2.70 & LysM domain-containing protein; VdSCP9 \\
\hline$V D A G \_01556$ & -2.39 & Neutral ceramidase \\
\hline$V D A G \_06199$ & -1.77 & SnodProt 1 \\
\hline$V D A G \_06993$ & -1.67 & $\begin{array}{l}\text { Necrosis- and ethylene-inducing protein } \\
\text { (NPP1-like) }\end{array}$ \\
\hline$V D A G \_04828$ & -1.65 & PRY1 protein \\
\hline$V D A G \_05456$ & -1.57 & Pisatin demethylase \\
\hline$V D A G \_01253$ & -1.52 & Plasma membrane fusion protein PRM1 \\
\hline
\end{tabular}
Predicted subcellular localization

Extracellular, soluble

Extracellular, soluble

Extracellular, soluble

Extracellular, soluble

Extracellular, soluble

Lysosome/vacuole, membrane

Extracellular, soluble

Extracellular, soluble

Extracellular, soluble

Extracellular, soluble

Extracellular, soluble

Extracellular, soluble

Extracellular, soluble

Extracellular, soluble

Extracellular, soluble

Extracellular, soluble

Extracellular, soluble

Extracellular, soluble

Extracellular, soluble

Extracellular, soluble

Extracellular, soluble

Extracellular, soluble

Extracellular, soluble

Extracellular, soluble

Extracellular, soluble

Extracellular, soluble

Extracellular, soluble

Extracellular, soluble

Extracellular, soluble

Extracellular, soluble

Extracellular, membrane

Peroxisome, soluble

Cytoplasm, soluble

Cytoplasm, soluble

Cytoplasm, soluble

Peroxisome, membrane

Cytoplasm, soluble

Cytoplasm, soluble

Peroxisome, soluble

Mitochondrion, soluble

Mitochondrion, membrane

Cytoplasm, soluble

Cytoplasm, soluble

Peroxisome, soluble

Cytoplasm, soluble

Extracellular, soluble

Extracellular, soluble

Cytoplasm, soluble

Extracellular, soluble

Cell membrane

Extracellular, soluble

Endoplasmic reticulum, membrane Endoplasmic reticulum, membrane

Endoplasmic reticulum, membrane

Extracellular, soluble

Extracellular, soluble

Extracellular, soluble

Extracellular, soluble

Extracellular, soluble

Endoplasmic reticulum, membrane

Cell membrane, membrane

(Continued on next page)

\footnotetext{
${ }^{a}$ Values represent $\log _{2}$ fold change. $\mathrm{WT}=$ wild type.

b Subcellular localization prediction: DeepLoc.

${ }^{c}$ Genes functionally characterized in this work by production of deletion mutants.
} 
Table 3. (Continued from previous page)

\begin{tabular}{|c|c|c|c|}
\hline Gene ID & WT vs. mutant ${ }^{a}$ & Functional annotation & Predicted subcellular localization ${ }^{b}$ \\
\hline$V D A G \_07321$ & -1.42 & Pisatin demethylase & Endoplasmic reticulum, membrane \\
\hline VDAG_02980 & -1.41 & Cell wall protein PhiA & Extracellular, soluble \\
\hline$V D A G \_01995$ & -1.38 & $25 \mathrm{kDa}$ protein elicitor; NPP1-like & Extracellular, soluble \\
\hline VDAG_08108 & -1.05 & Hce2 family, pathogen effector & Extracellular, soluble \\
\hline \multicolumn{4}{|l|}{ Protease genes } \\
\hline VDAG_00499 & -5.06 & Endothiapepsin & Extracellular, soluble \\
\hline$V D A G \_00500$ & -4.85 & Carboxypeptidase S1 & Extracellular, soluble \\
\hline$V D A G \_07273$ & -3.23 & Leucyl aminopeptidase & Extracellular, soluble \\
\hline$V D A G \_08867$ & -2.79 & Carboxypeptidase & Extracellular, soluble \\
\hline$V D A G \_05635$ & -2.70 & Peptidase S15 & Peroxisome, soluble \\
\hline$V D A G \_07176$ & -2.41 & Alkaline proteinase & Extracellular, soluble \\
\hline$V D A G \_07183$ & -2.30 & Carboxypeptidase A & Extracellular, soluble \\
\hline$V D A G \_08100$ & -2.29 & Serin endopeptidase & Extracellular, soluble \\
\hline$V D A G \_00129$ & -2.11 & Metalloproteinase & Extracellular, soluble \\
\hline$V D A G \_03905$ & -2.02 & Serin endopeptidase & Cytoplasm, soluble \\
\hline$V D A G \_04551$ & -1.99 & Antigen 1 M35-like peptidase & Extracellular, soluble \\
\hline$V D A G \_10297$ & -1.82 & Peptidase M28 & Extracellular, soluble \\
\hline$V D A G \_03555$ & -1.82 & Thymus-specific serine protease & Extracellular, soluble \\
\hline$V D A G \_05652$ & -1.73 & Hypothetical protein & Extracellular, soluble \\
\hline$V D A G \_06386$ & -1.71 & Tripeptidyl-peptidase & Extracellular, soluble \\
\hline$V D A G \_09397$ & -1.48 & Dipeptidyl aminopeptidase & Extracellular, soluble \\
\hline$V D A G \_00795$ & -1.41 & Zinc carboxypeptidase A 1 & Lysosome/vacuole \\
\hline$V D A G \_07103$ & -1.40 & Ubiquitin/metalloprotease fusion protein & Nucleus, soluble \\
\hline$V D A G \_01706$ & -1.33 & Aminopeptidase $\mathrm{Y}$ & Cytoplasm, soluble \\
\hline$V D A G \_03907$ & -1.21 & Aminopeptidase Y & Extracellular, soluble \\
\hline$V D A G \_02973$ & -1.03 & Conserved hypothetical protein & Extracellular, soluble \\
\hline$V D A G \_07980$ & -1.01 & Aminopeptidase $\mathrm{Y}$ & Extracellular, soluble \\
\hline \multicolumn{4}{|c|}{ Genes encoding signaling pathway components and proteins with regulatory functions } \\
\hline$V D A G \_00683(V d R G S 1)^{\mathrm{c}}$ & 3.38 & Developmental regulator FlbA & Cytoplasm, soluble \\
\hline$V D A G \_08032$ & 1.84 & Snf2 helicase-like protein & Nucleus, soluble \\
\hline$V D A G \_00674$ & 1.61 & Akyrin repeats and SAM-domain & Cytoplasm, soluble \\
\hline$V D A G \_05860$ & 1.52 & Homeobox transcription factor (Pth12) & Nucleus, soluble \\
\hline$V D A G \_03447$ & 1.25 & $\begin{array}{l}\text { HLyIII_domain G protein coupled receptors } \\
\text { (GPCR) }\end{array}$ & Cell membrane, membrane \\
\hline$V D A G \_07045$ & 1.23 & $\begin{array}{l}\text { Similar to regulator of } \mathrm{G} \text { protein signaling } \\
\text { proteins }\end{array}$ & Endoplasmic reticulum, membrane \\
\hline$V D A G \_00675$ & 1.20 & Putative protein kinase & Cytoplasm, soluble \\
\hline$V D A G \_03091$ & 1.15 & Similar to Hex $2 / \operatorname{Reg} 1$ & Nucleus, soluble \\
\hline$V D A G \_00047$ & 1.01 & Snf2 helicase-like protein & Nucleus, soluble \\
\hline$V D A G \_05156$ & -2.68 & $\mathrm{C}_{2} \mathrm{H}_{2}$ zinc finger protein & Nucleus, soluble \\
\hline$V D A G \_05213$ & -2.66 & $\mathrm{C}_{2} \mathrm{H}_{2}$ zinc finger protein & Nucleus, soluble \\
\hline$V D A G \_00541$ & -1.97 & Family C-like GPCR & Cell membrane \\
\hline$V D A G \_07039(v r g 1)^{\mathrm{c}}$ & -1.96 & Ras-like GTPase domain & Mitochondrion, membrane \\
\hline$V D A G \_05713$ & -1.79 & Related to Pth11 & Cell membrane \\
\hline$V D A G \_06955$ & -1.58 & Pth11-like & Cell membrane \\
\hline$V D A G \_01858(v s s l)^{\mathrm{c}}$ & -1.20 & Virulence sensor protein bvgS & Cytoplasm, soluble \\
\hline \multicolumn{4}{|l|}{ Transporter genes } \\
\hline$V D A G \_03237$ & 4.39 & Maltose permease MAL31 & Lysosome/vacuole, membrane \\
\hline$V D A G \_09071$ & 1.53 & High-affinity glucose transporter RGT2 & Cell membrane \\
\hline$V D A G \_06278$ & 1.48 & Siderophore iron transporter mirB & Cell membrane \\
\hline$V D A G \_09374$ & 1.46 & Pantothenate transporter liz1 & Endoplasmic reticulum, membrane \\
\hline$V D A G \_02710$ & 1.42 & High-affinity glucose transporter SNF3 & Cell membrane \\
\hline VDAG_09734 & 1.24 & Major myo-inositol transporter iolT & Cell membrane \\
\hline$V D A G \_06939$ & 1.22 & Proton myo-inositol cotransporter & Cell membrane \\
\hline$V D A G \_09282$ & 1.07 & Proton myo-inositol cotransporter & Cell membrane \\
\hline$V D A G \_07186$ & -3.22 & Ammonium transporter & Cell membrane \\
\hline$V D A G \_09702$ & -2.63 & Sugar transporter STL1 & Cell membrane \\
\hline$V D A G \_03698$ & -2.19 & Polyamine transporter 1 & Cell membrane \\
\hline$V D A G \_06373$ & -2.18 & Similar to GABA permeases & Cell membrane \\
\hline$V D A G \_03336$ & -2.00 & Excitatory amino acid transporter 2 & Cell membrane \\
\hline$V D A G \_08061$ & -2.00 & SGE1 (VBA5) & Cell membrane \\
\hline$V D A G \_02849$ & -1.86 & Polyamine transporter 3 & Cell membrane \\
\hline$V D A G \_08084$ & -1.83 & Oligopeptide transporter & Endoplasmic reticulum, membrane \\
\hline$V D A G \_04887$ & -1.79 & Dicarboxylic amino acid permease & Endoplasmic reticulum, membrane \\
\hline$V D A G \_06062$ & -1.77 & Proline-specific permease & Endoplasmic reticulum, membrane \\
\hline$V D A G \_07108$ & -1.73 & Proline-specific permease & Cell membrane \\
\hline$V D A G \_10208$ & -1.61 & Amino-acid permease inda1 & Endoplasmic reticulum, membrane \\
\hline$V D A G \_02764$ & -1.58 & Urea active transporter (Dur3) & Cell membrane \\
\hline$V D A G \_07042$ & -1.44 & Polyamine transporter 1 & Cell membrane \\
\hline$V D A G \_10467$ & -1.40 & Dicarboxylic amino acid permease & Endoplasmic reticulum, membrane \\
\hline$V D A G \_02246$ & -1.11 & Polyamine transporter 3 & Cell membrane \\
\hline VDAG_01105 & -1.01 & Polyamine transporter 1 & Cell membrane \\
\hline
\end{tabular}


above. For example, VDAG_06199, which was 3.4-fold more highly expressed in the random mutant than in the WT, encodes a SnodProt1-like secreted protein shown to play a role in virulence in $V$. dahliae (Zhang et al. 2017). Secreted proteases are also part of the secretome and might function as virulence factors. A total of 22 differentially expressed protease-encoding genes were identified in our analysis; interestingly, all of them were upregulated in the random mutant strain. Most of these proteases were predicted to be secreted proteins (Table 3). The adjacent protease genes, VDAG_00499 and VDAG_00500, exhibited the highest levels of upregulation in mutant versus WT, with $\log _{2} \mathrm{FC}$ values of 5.06 and 4.85 , respectively. VDAG_00499 is very similar to aspegillopepsin $\mathrm{F}$, a secreted aspartic protease involved in host invasion in Aspergillus fumigatus (Lee and Kolattukudy 1995). VDAG_04551, encoding a putative homolog of the protease Pral from Candida albicans, was also induced in the mutant. Pra1 is involved in host recognition and its absence reduces adhesion to host cells (Soloviev et al. 2011). Genes encoding two pisatin demethylases, two necrosis- and ethyleneinducing proteins (NPP1-like), and an effector from the Hce2 family were all up-regulated in the mutant (Table 3). Taken together, our results show that a number of activities potentially associated with virulence are induced in the random mutant D$10-8 \mathrm{~F}$.

\section{Genes potentially associated with signal transduction pathways and regulatory functions exhibit differential expression in the WT versus random mutant strain.}

Several genes encoding putative components of signal transduction pathways were differentially expressed in the WT versus D-10-8F (Table 3). Zheng et al. (2010) have identified genes predicted to encode $G$ protein coupled receptors (GPCRs) in $V$. dahliae in a genome-wide analysis. One of these genes, VDAG_00541, encoding a family C-like GPCR, exhibited significant levels of upregulation in the mutant, whereas VDAG_03447, a HLyIII_domain GPCR gene, was 2.4fold more highly expressed in the WT than in the mutant strain. A putative Ras GTPase gene,VDAG_07039, and VDAG_07039 and $V D A G \_06955$, encoding Pth11-like proteins, which are related to GPCRs (Kulkarni et al. 2005), were all more expressed in D-10-8F than in the WT. GPCRs and regulators of $\mathrm{G}$ protein signaling (RGS) proteins function as activators and repressors of $\mathrm{G}$ protein signaling, respectively. Very interestingly, VDAG_00683, one of the genes with the highest levels of upregulation in the WT $\left(\log _{2} \mathrm{FC}=3.38\right)$, encodes a putative homolog of the RGS protein FlbA, which plays important roles in growth and development in fungi. A second gene encoding a putative RGS protein, $V D A G \_07045$, exhibited moderate induction in the WT. VDAG_03091, similar to $S$. cerevisiae hex2/reg1, also exhibited higher levels of expression in the WT than in the mutant strain. Hex $2 / \operatorname{Reg} 1$ is the regulatory subunit of the phosphatase CID1/GLC7. In $S$. cerevisiae, mutants deleted for hex2 display constitutive activation of cAMP signaling (Dumortier et al. 1995). $V D A G \_07186$, encoding an ammonium transporter highly similar to Mep2, by contrast, was strongly up-regulated in the D-10-8F strain. Mep2 transporters have been implicated in the mitogen-activated protein kinase (MAPK) and cAMP signaling pathways (Biswas and Morschhäuser 2005; Lorenz and Heitman 1998). Finally, VDAG_01858, which encodes a hybrid

Table 4. Putative secondary metabolism gene clusters from Verticillium dahliae identified as upregulated in random T-DNA insertion mutant D-10-8F versus the wild type (WT)

\begin{tabular}{|c|c|c|}
\hline Gene ID & Mutant vs. WT ${ }^{\mathbf{a}}$ & Functional annotation \\
\hline$V D A G \_00900$ & 3.55 & Predicted protein \\
\hline VDAG_00901 & 2.90 & Killer toxin subunits $\alpha / \beta$ \\
\hline$V D A G \_00902$ & 2.70 & LysM domain-containing protein (VdSCP9) \\
\hline$V D A G \_02062$ & 4.36 & Translation initiation inhibitor \\
\hline$V D A G \_02063$ & 5.52 & L-amino-acid oxidase \\
\hline$V D A G \_02064$ & 2.93 & Conserved hypothetical protein \\
\hline$V D A G \_02065$ & 2.95 & TRI14 \\
\hline$V D A G \_02909$ & 2.16 & Conserved hypothetical protein \\
\hline$V D A G \_02910$ & 1.71 & Enoyl-CoA hydratase/isomerase \\
\hline$V D A G \_02911$ & 2.77 & Surfactin synthetase subunit 3 \\
\hline$V D A G \_02912$ & 0.67 & C6 zinc finger domain-containing protein \\
\hline$V D A G \_02913$ & 1.66 & Brefeldin A resistance protein \\
\hline$V D A G \_05830$ & 1.13 & Cytochrome P450 2C31 \\
\hline$V D A G \_05831$ & 4.05 & Phenylalanine ammonia-lyase \\
\hline$V D A G \_05832$ & nd & FAD binding domain-containing protein \\
\hline$V D A G \_05833$ & 5.02 & Conserved hypothetical protein \\
\hline$V D A G \_05834$ & 5.89 & Conserved hypothetical protein \\
\hline$V D A G \_05835$ & nd & Aldehyde reductase \\
\hline$V D A G \_05836$ & 4.74 & Parahydroxybenzoate-polyprenyltransferase \\
\hline$V D A G \_05837$ & 4.21 & FAD binding domain-containing protein \\
\hline$V D A G \_05838$ & 2.90 & $\begin{array}{l}\text { Alcohol dehydrogenase GroES domain- } \\
\text { containing protein }\end{array}$ \\
\hline$V D A G \_05839$ & 2.82 & Conserved hypothetical protein \\
\hline$V D A G \_09522$ & 1.40 & Aquaporin-9 \\
\hline$V D A G \_09523$ & 2.12 & Pectate lyase D \\
\hline$V D A G \_09524$ & 1.76 & Dibenzothiophene desulfurization enzyme C \\
\hline$V D A G \_09525$ & nd & Accumulation of DYads \\
\hline$V D A G \_09526$ & 7.18 & FAD binding domain-containing protein \\
\hline$V D A G \_09527$ & 6.14 & Hydrolase \\
\hline$V D A G \_09528$ & 1.18 & Conserved hypothetical protein \\
\hline$V D A G \_09529$ & 2.06 & Conserved hypothetical protein \\
\hline$V D A G \_09530$ & nd & Conserved hypothetical protein \\
\hline$V D A G \_09531$ & 4.78 & Predicted protein \\
\hline$V D A G \_09532$ & 4.79 & Serine 3-dehydrogenase \\
\hline$V D A G \_09533$ & nd & Conserved hypothetical protein \\
\hline$V D A G \_09534$ & 4.89 & Aflatoxin biosynthesis polyketide synthase \\
\hline
\end{tabular}

\footnotetext{
${ }^{\text {a }}$ Values shown as $\log _{2}$ foldchange; nd = not differentially expressed.
} 
histidine kinase (HHK) similar to the virulence sensor protein BvgS (Uhl and Miller 1996), was up-regulated 2.3-fold in the mutant.

Transcription factors (TFs) are major targets of signaling pathways connecting extracellular signals to the transcriptional changes required to adaptive responses. Few genes encoding proteins exhibiting clear homologies with known TFs were identified as differentially expressed in our analysis. Interestingly, however, $16.5 \%$ of the 206 genes exhibiting higher levels of expression in WT than in the random mutant encoded predicted nuclear proteins. In contrast, only $5.3 \%$ of the 366 genes upregulated in the mutant were predicted to encode nuclear proteins. Genes identified as upregulated in WT predicted to encode nuclear proteins include VDAG_05860, a putative homolog of the homeobox TF gene $M o H O X 7$, required for virulence in Magnaporthe oryzae (Kim et al. 2009). $V D A G \_00047$, encoding a large protein potentially involved in DNA methylation and also containing a helicase domain, exhibited a twofold increase in expression in the WT versus the random mutant. A second putative helicase-like gene, $V D A G \_08032$, was similarly up-regulated in the WT.

\section{Deletion mutants lacking $V D A G \_00683$ (VdRGS1) exhibit phenotypic alterations that are indistinguishable from those observed in random mutant D-10-8F.}

$V D A G \_00683$, highly upregulated in the WT versus random mutant, was the first differentially expressed gene selected for further characterization. As stated, VDAG_00683 is a putative homolog of the RGS protein FlbA, which plays important roles in growth and development in fungi. Recently, Xu et al. (2018) provided evidence that this RGS protein, which they termed VdRGS1, is involved in pathogenicity in V. dahliae and used it as a target of plant-induced gene silencing. However, its potential implication in the signaling pathways regulating $V$. dahliae morphogenesis and specific role in virulence were not investigated. We hypothesized that the strong repression of VdRGS1 observed in strain D-10-8F could be an important factor contributing to either its reduced virulence, alterations in polar growth and MS production, or both. To test our hypothesis, the open reading frame (ORF) of VdRGS1 was deleted in the parental strain of random mutant D-10-8F, V. dahliae DvdT5 (Dobinson et al. 1996). For that, we used the OSCAR method (Gold et al. 2017; Paz et al. 2011) but replaced the binary vector pOSCAR by pOSCAR-HSVtk, which contains the toxic gene HSVtk (herpes simplex virus thymidine kinase) between the BP clonase recombination site P3 and the T-DNA right border (Fig. 4). HSVtk is a negative selection marker in the presence of 5-fluoro-2'-deoxyuridine (5FU), which is converted by $H S V t k$ product to a compound toxic in fungal species (Khang et al. 2005). Transformants in which deletion constructs generated with pOSCAR-HSVtk integrate ectopically contain the toxic gene HSVtk and are selected against in the presence of $5 \mathrm{FU}$, whereas integration by homologous recombination of the target gene flanks prevents integration of HSVtk in the fungal genome (Fig. 4). By preventing ectopic integration of the T-DNA, pOSCAR-HSVtk greatly simplifies the selection of deletion mutants. This modified version of the OSCAR methodology has been tested, for the first time, in this work to produce VdRGS1 deletion mutants. PCR analysis of individual transformants indicated that HSVtk worked well, preventing random integration of the deletion construct T-DNA; all 10 transformants tested lacked the VdRGSI ORF. Two independent deletion mutants, designated $\Delta v d r g s 1-1$ and $\Delta v \operatorname{drgs} 1-2$, were selected for phenotypic characterization, after confirming by Southern blot analysis that they harbored no secondary insertions of the T-DNA that could be affecting other genes (Supplementary Fig. S1).
Strains $\Delta v d r g s 1-1$ and $\Delta v d r g s 1-2$ were found to closely resemble random mutant D-10-8F, exhibiting a drastic reduction in MS formation, both on PDA plates and in liquid Czapek medium (Fig. 5A and B). We then examined if the $\Delta v d r g s 1-1$ and $\Delta v d r g s 1-2$ strains also shared the alteration in polar growth observed in D-10-8F grown on PDA covered with cellophane. In order to perform morphology comparisons in identical conditions, D-10-8F and the WT were included along with $\Delta v d r g s 1-1$ and $\Delta v d r g s 1-2$ in this analysis. Microscopic observations were performed on strains grown on PDA covered with cellophane for a period of 48 to $96 \mathrm{~h}$. It was found that the hyperpolarized growth of $\Delta v d r g s 1-1$ and $\Delta v d r g s 1-2$ was indistinguishable from that of D-10-8F; all three strains produced bundles of hyphae that exhibited very little branching, growth which contrasted with the profuse branching and swollen hyphal tips observed in the WT (Fig. 5C). Finally, we performed virulence assays using $N$. benthamiana as a host to examine the ability of $\Delta v d r g s 1-1$ and $\Delta v d r g s 1-2$ to induce Verticillium wilt symptoms. The random mutant D-10-8F was included in these assays, alongside the two deletion mutants, to be able to compare disease symptoms within the same experiments. There was a statistically significant reduction in disease severity when plants were inoculated with $\Delta v d r g s 1-1$ or $\Delta v d r g s 1-2$ as compared with the WT infection. Furthermore, the severity of Verticillium wilt symptoms induced by D-10-8F and the two deletion mutants were not statistically different (Fig. 6).

\section{Deletion of $V D A G \_07039$ (vrg1) or VDAG_01858 (vvs1) does not have discernible phenotypic effects.}

$V D A G \_07039$ and VDAG_01858 were selected as the initial candidates for functional characterization among those genes exhibiting higher levels of expression in the random mutant than in the WT. The annotated protein VDAG_07039 exhibits similarity to Ras-like GTPases, whereas VDAG_01858 is a putative HHK. HHKs are "two-component" system sensor kinases that have been associated with different roles in plantpathogenic fungi (Hérivaux et al. 2016). We hypothesized that $V D A G \_07039$ and $V D A G \_01858$ differentially expressed genes, as they encode proteins with regulatory functions, might play important roles in $V$. dahliae. To delete these genes, which we designated vrg1 (VDAG_07039) and vvs1 (VDAG_01858), the same modified version of the OSCAR method developed to produce $\Delta v d r g s 1-1$ and $\Delta v d r g s 1-2$ was used (Fig. 4). A PCR analysis confirmed deletion of $v r g l$ in all 10 transformants tested. Two of the deletion mutants, designated $\Delta v \mathrm{rg} 1-1$ and $\Delta v r g 1-2$, were further characterized, confirming gene deletion and absence of secondary insertions of the T-DNA, by Southern blot analysis (Supplementary Fig. S2) and were selected for phenotypic characterization. Similarly, deletion of $v v s 1$ was confirmed in nine of 10 transformants analyzed by PCR, and two of the deletion mutants, designated $\Delta v v s 1-1$ and $\Delta v v s 1-2$, were further characterized by Southern blot analysis (Supplementary Fig. S3) and were selected for phenotypic characterization. Strains $\Delta v v s 1-1 / \Delta v v s 1-2$ and $\Delta v \operatorname{rg} 1-1 / \Delta v r g 1-2$ did not exhibit phenotypic alterations when grown on solid or in liquid medium, nor were they affected in their ability to induce Verticillium wilt symptoms (Supplementary Fig. S4).

\section{DISCUSSION}

Analysis of the molecular mechanisms regulating development and virulence in plant-pathogenic fungi can provide potential targets for disease control. In this work, we characterized a strain, generated by T-DNA insertional mutagenesis, exhibiting alterations in growth and reduced virulence by comparing its global transcription pattern with that of the WT strain. In this way, we expected to identify the genes, molecular mechanisms, 
and cellular processes that were altered in this random mutant and that could, therefore, be involved in virulence and development in $V$. dahliae.

As in other eukaryotes, $\mathrm{G}$ protein-mediated signaling plays major roles in filamentous fungi ( $\mathrm{Li}$ et al. 2007). Each heterotrimeric $G$ protein is composed of subunits $G \alpha, G \beta$, and $G \gamma$ bound to the plasma membrane. In the inactive state, the three subunits form a complex associated with a GPCR. Binding of a ligand to the GPCR results in the exchange of GTP for GDP in the $G \alpha$ subunit, and this leads to its dissociation from the $G \beta \gamma$ heterodimer. The dissociated active form of the $G$ protein regulates downstream effector proteins in various signaling pathways, including cAMP signaling and MAPK cascades ( $\mathrm{Li}$ et al. 2007). In $V$. dahliae functional analyses of $V d P K C l$, encoding the catalytic subunit of a cAMP dependent protein kinase $\mathrm{A}$, and $V G B$, the $\mathrm{G}$ protein $\beta$-subunit gene, have provided evidence that they are negative regulators of MS formation and positive regulators of virulence, likely functioning in the same pathway (Tzima et al. 2010; 2012). Interestingly, one of the genes with the highest levels of upregulation in the WT versus random mutant D-10-8F encodes the RGS protein VdRGS1. RGS proteins play a negative role in the regulation of G proteins by promoting the hydrolysis of GTP to GDP by the

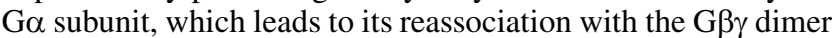
(Li et al. 2007). VdRGS1 is a putative homolog of FlbA, a wellcharacterized RGS protein from Aspergillus nidulans. There is evidence that FlbA blocks proliferation and induces development through the repression of $\mathrm{G}$ protein signaling in A. nidulans (Yu et al. 1996). The identification of VdRGS1 as highly downregulated in a mutant exhibiting hyperpolarized growth and clearly impaired in the formation of MS and virulence indicated that the FlbA homolog in $V$. dahliae might have a similar role. That is, to downregulate $G$ protein signaling to block fungal proliferation and induce MS development, allowing completion of the disease cycle. VdRGS1 has, indeed, been shown to be required for full virulence and has been used as a target for host-induced gene silencing (Xu et al. 2018). However, its implication in the signaling pathways that

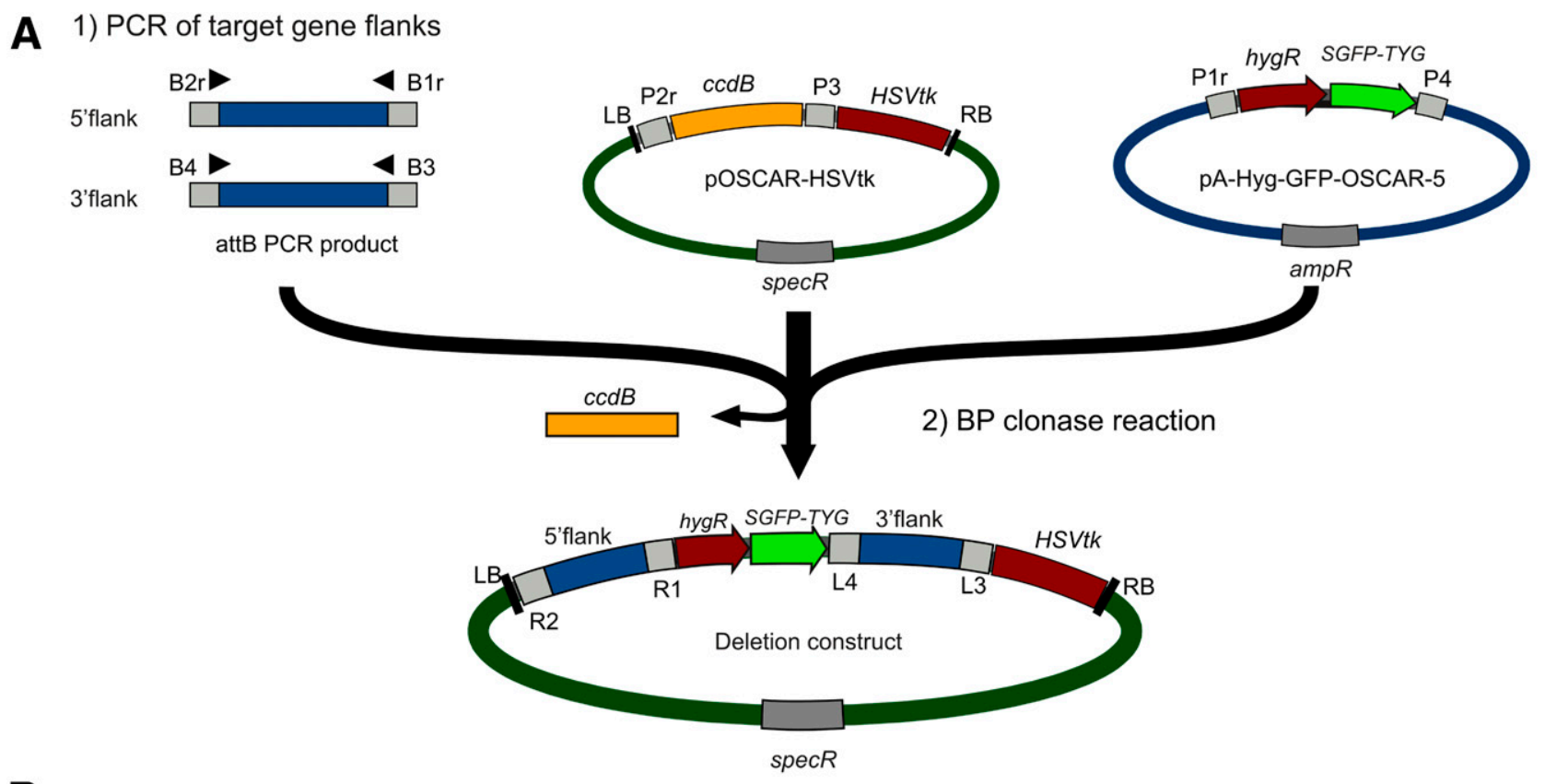

B

Integration of T-DNA by homologous recombination of target gene flanks: deletion mutant

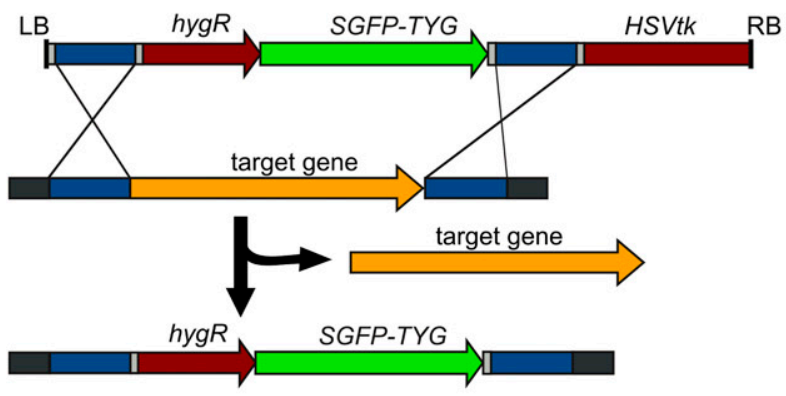

Random integration of T-DNA in the fungal genome

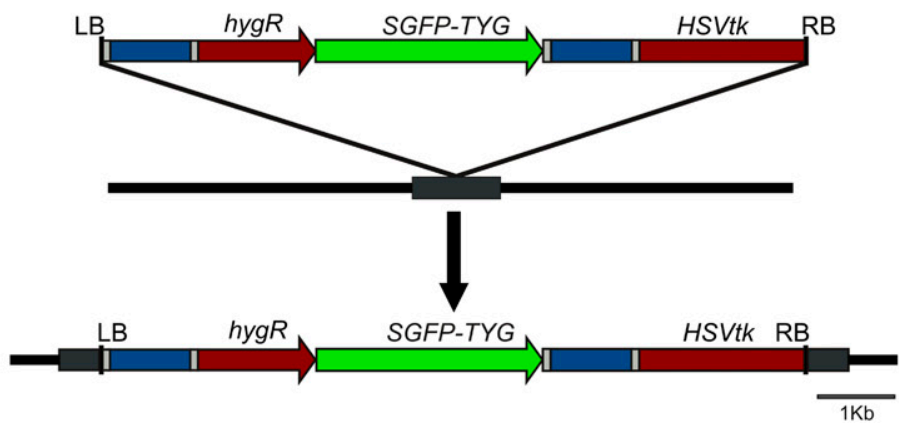

Fig. 4. Production of deletion mutants in Verticillium dahliae, using a modified version of one-step construction of Agrobacterium-recombination-ready plasmids (OSCAR). A, To produce deletion mutants, the OSCAR method (Paz et al. 2011) was used but vector pOSCAR was replaced with a new version containing the toxic gene HSVtk (herpes simplex virus thymidine kinase). To construct the new vector, termed pOSCAR-HSVtk, the HSVtk cassette was amplified from plasmid pGKO2 (Khang et al. 2005), introducing HindIII recognition sequences at the 5' ends, and was then cloned into the unique HindIII site of pOSCAR. B, Upon transformation of the fungus with deletion constructs generated with pOSCAR-HSVtk, only deletion mutants are generally selected in the presence of 5-fluoro-29-deoxyuridine (5FU), as those transformants in which the T-DNA is randomly inserted contain HSVtk, whose product converts 5FU to a compound toxic to the fungus. This modified version of OSCAR was used to delete genes VdRGS1 (VDAG_00683), vrg1 (VDAG_07039), and vvs1 (VDAG_01858). 
governed development and virulence in $V$. dahliae had not been examined. We found that deletion of VdRGS1 resulted in phenotypic alterations in polar growth, MS production, and virulence that were indistinguishable from that of the random mutant D-10-8F. These results confirmed that the phenotypic alterations observed in $\mathrm{D}-10-8 \mathrm{~F}$ resulted from the disruption of VdRGS1 activity.

In Aspergillus niger deletion of flbA results in a more complex secretome (Krijgsheld et al. 2013). Interestingly, our results showed that $30 \%$ of the proteins encoded by the genes upregulated in the random mutant D-10-8F versus WT were predicted to be secreted, indicating that absence of VdRGS1 activity might have a similar impact in increasing secretome complexity in $V$. dahliae. For instance, a number of genes predicted to encode extracellular proteases exhibited induction in D-10-8F versus WT. Secreted proteases play relevant roles during pathogenic development; they can facilitate invasion by degrading host tissue, destroy plant defense molecules, and digest proteins for nutrient supply in conditions in which other nitrogen sources might be scarce (Naglik et al. 2004).

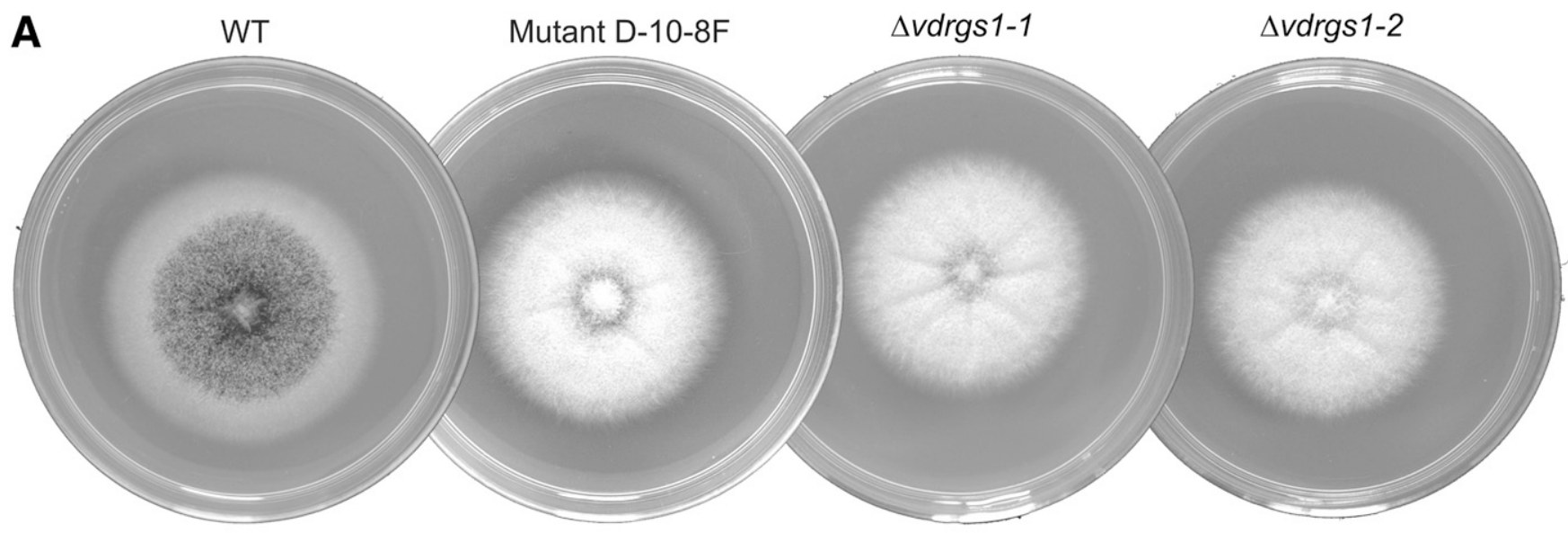

B

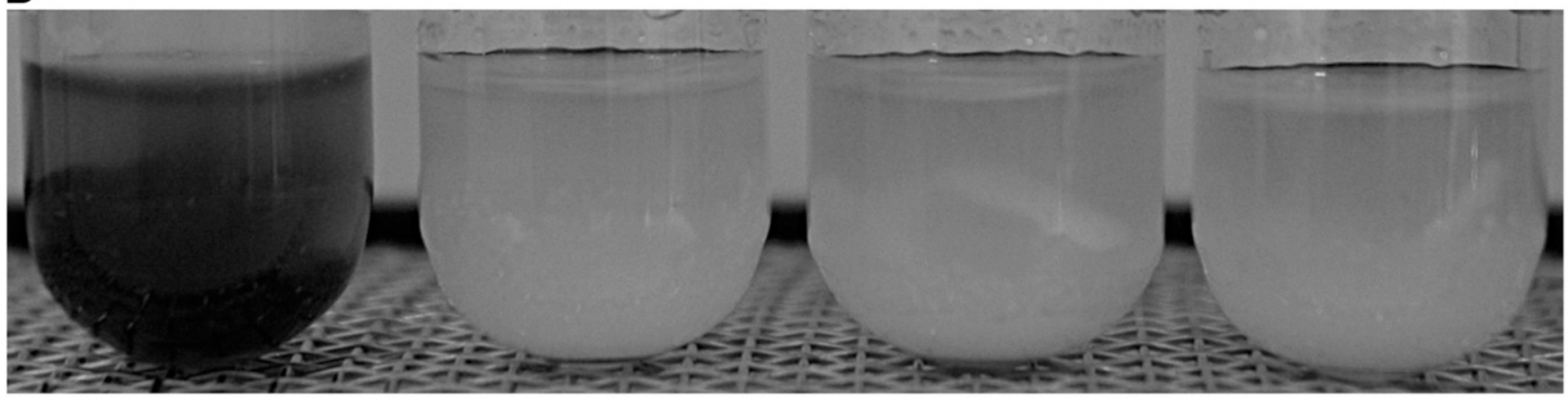

C

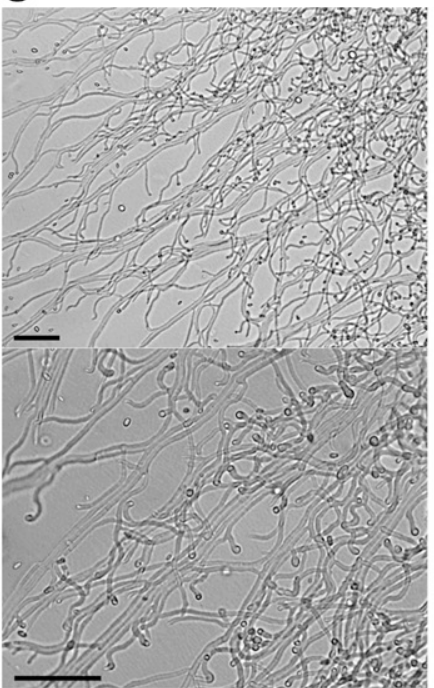

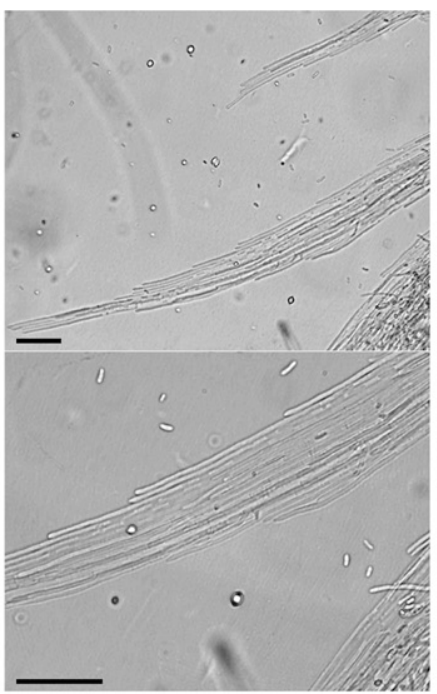
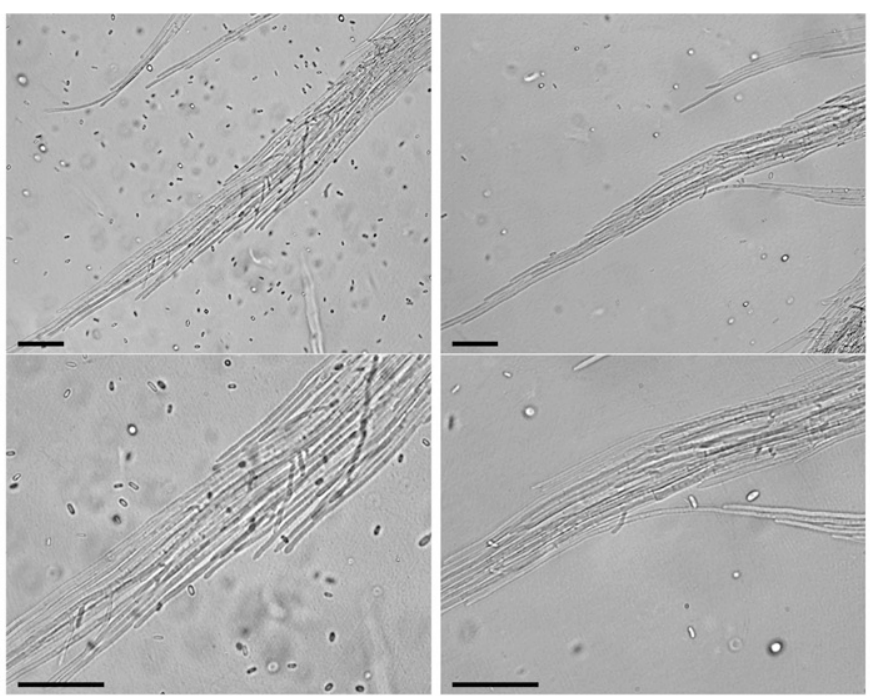

Fig. 5. Verticillium dahliae deletion mutants lacking VdRGS1 and random T-DNA insertion mutant D-10-8F exhibit very similar morphological alterations. A, Colony morphology of the wild type (WT), random mutant D-10-8F, and strains $\Delta v \operatorname{drgs} 1-1$ and $\Delta v \operatorname{drgs} 1-2$, grown for 12 days on potato dextrose agar (PDA) at $24^{\circ} \mathrm{C}$. B, Strains grown in Czapek Dox broth for 7 days at $24^{\circ} \mathrm{C}$ and $200 \mathrm{rpm}$. C, Microscopic observations of strains grown on PDA covered with cellophane for 3 days. Scale bar $=50 \mu \mathrm{m}$. 
$V D A G \_07186$, highly similar to the ammonium transporter gene $m e p 2$, was also highly induced in the mutant, as compared with its expression in WT. Mep2 proteins can have signaling functions in addition to their role as transporters. They have been characterized as upstream components of the cAMP and MAPK signaling pathways, linking nutrient availability sensing with the regulation of fungal development and virulence (Paul et al. 2018; Rutherford et al. 2019). In Candida albicans, there is a well-characterized coregulation of the expression of mep2 and the secreted aspartic protease gene sap 2 that also involves the induction of transporters of the OPT (oligopeptide transporter) family (Morschhäuser 2011). We identified a putative homolog of mep2 (VDGA_07186), other nitrogen compound transporter genes, including $V D A G \_08084$ of the OPT family, and numerous secreted protease genes, including VDGA_00499 encoding a secreted aspartic protease, all of them were upregulated in random mutant D10-8F versus WT. VDGA_00499 and VDGA_07186 were both highly differentially expressed, with $\log _{2} \mathrm{FC}$ values of 3.22 and 5.06 , respectively. This
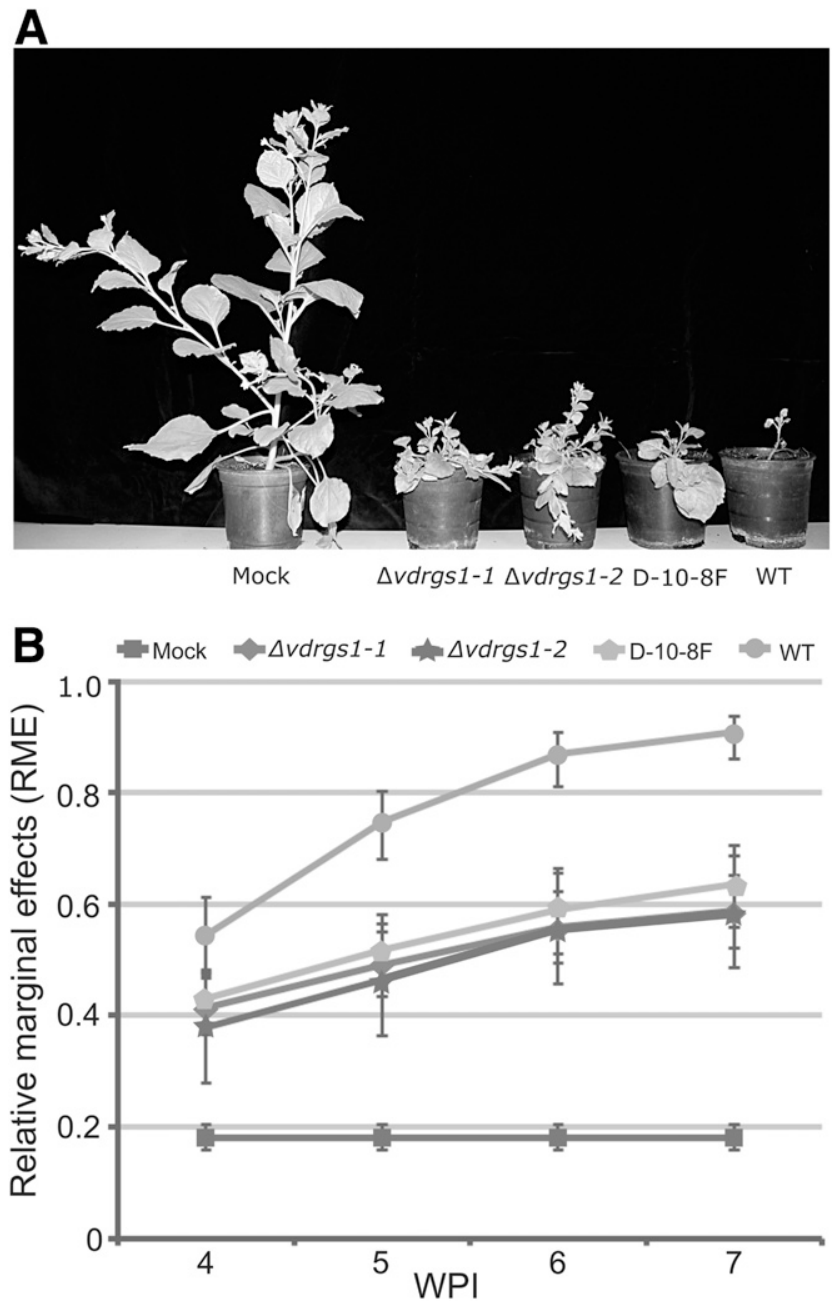

Fig. 6. Verticillium dahliae deletion mutants lacking VdRGS1 and random T-DNA insertion mutant D-10-8F are similarly affected in their ability to induce Verticillium wilt symptoms. A, Nicotiana benthamiana plants showing reduced Verticillium wilt symptoms in infections with mutant strains as compared with wild-type (WT) infection; control plants were mock inoculated with water. B, Relative marginal effects (RME) disease severity over a period of 7 weeks, calculated according to a 0 to 3 scale: $0=$ healthy leaf, 1 = chlorotic leaf, 2 = leaf with wilt symptoms, and $3=$ fully necrotic leaf. Bars are lower and upper confident intervals (CIs) at $95 \%$ probability. Two treatments were considered different when the CIs did not overlap. transcription pattern suggests that, in $V$. dahliae, expression of mep 2 and secreted protease genes could be coordinated. Changes in carbon and nitrogen metabolism are known to be associated with virulence in V. dahliae (Klimes et al. 2015). To confirm the presence of a molecular mechanism that links the sensing of specific nitrogen nutritional conditions encountered by $V$. dahliae on plant tissues to the induction of virulence factors such as secreted proteases would be of great interest.

Other relevant features of the random mutant transcriptome potentially associated with responses to nutritional conditions include the upregulation of genes encoding key enzymes of the GABA shunt. The GABA produced by plants as a stress response could be uptaken and metabolized by phytopathogenic fungi during infection (Bönnighausen et al. 2015; Solomon and Oliver 2002). In the so-called GABA shunt, GABA transaminases convert GABA to succinic semialdehyde, which is then further oxidase to succinate by SSADHs. Disruption of the GABA shunt in $F$. graminearum by deletion of its two GABA transaminases, $g t a_{1}$ and $g t a_{2}$, resulted in reduced virulence (Bönnighausen et al. 2015). We found that VDAG_06397 and $V D A G \_04571$, putative homologs of $g t a_{1}$ and $g t a_{2}$, respectively, were up-regulated in D-10-8F versus WT. Furthermore, two SSADH genes, VDAG_03345 and VDAG_04572, were both roughly 30 -fold more expressed in the mutant than in the WT strain. PAs also accumulate in plants in response to pathogen infection (Valdés-Santiago and Ruiz-Herrera 2014; ValdésSantiago et al. 2012). Bönnighausen et al. (2015) have proposed that $F$. graminearum uptakes plant putrescine and metabolizes it through the GABA shunt during wheat head colonization. We found that genes encoding oxidases potentially involved in the synthesis of GABA from putrescine were up-regulated in D-108F. What is more, VDAG_02764 encoding a protein highly similar to the transporter Dur3 involved in the uptake of putrescine and a whole set of genes encoding transporters exhibiting similarity to Tpo1-Tpo4, which are involved in the excretion of PAs (Igarashi and Kashiwagi 2010; ValdésSantiago et al. 2012), were all up-regulated in D-10-8F versus WT. Genes encoding glutamate decarboxylase, which produce GABA from glutamate, or key PA biosynthetic enzymes, such as ornithine decarboxylase and $S$-adenosylmethionine decarboxylase (Valdés-Santiago and Ruiz-Herrera 2014), were not identified as differentially expressed in our analysis. These results show that endogenous GABA production and PA synthesis were not up-regulated in the $V$. dahliae random mutant D-10-8F. Mo et al. (2015) confirmed the important role of PAs in the defense responses of cotton to $V$. dahliae infection. The transcription pattern described here for mutant D-10-8F could be associated with an in-planta response, suggesting that $V$. dahliae copes with PAs produced by the host by metabolizing putrescine through the GABA shunt and inducing the expression of transporters involved in their excretion. In this way the fungus would maintain intracellular concentration of PAs that are not toxic and allow normal fungal development and progression of the disease cycle.

The predicted functions of other differentially expressed genes points in the same direction, that what we are observing in the random mutant D-10-8F is the induction of activities normally associated with pathogenic development; for example, genes predicted to encode secreted pectinolytic enzymes were identified as upregulated in this mutant strain versus their WT expression. Pectin is a major component of the primary plant cell wall and middle lamella. Pectinases may have particular importance as virulence factors in vascular wilt pathogens, as infected xylem vessels are occluded by pectin gels (Reignault et al. 2008). The genome of $V$. dahliae has been found, in fact, to encode more pectinolytic enzymes than fungal pathogens with other lifestyles (Klosterman et al. 2011). 
Recently, Yang et al. (2018) showed that the V. dahliae pectate lyase VdPEL1 plays a role in virulence and induces plant immune responses. In our analysis, four pectate lyase and two pectin lyase genes were all up-regulated in the mutant strain. A pectinesterase gene (VDAG_07881) and an endopolygalacturonase gene (VDAG_04977) were also among the pectinolytic genes whose expression was strongly induced in the mutant.

Several other individual genes that have been functionally characterized in $V$. dahliae and encode virulence factors were up-regulated in D-10-8F. These include VDAG_06199, encoding a SnodProt1-like secreted protein (Zhang et al. 2017), and $V D A G \_00902$, encoding a putative effector protein with a LysM domain (VdSCP9) Zhou et al. (2017). Toxins produced by vascular wilt pathogens are frequently associated with the induction of disease symptoms (Yadeta and Thomma 2013). The expression of NLP2 (VDAG_01992), which codes for one of the two necrosis- and ethylene-inducing proteins displaying cytotoxic activity in $V$. dahliae (Santhanam et al. 2013; Zhou et al. 2012), was among the genes identified as induced in the random mutant strain D-10-8F. Several secondary metabolism gene clusters potentially involved in the production of phytotoxins also exhibited higher levels of expression in the mutant than in WT. For example, a cluster that encodes a protein similar to TRI14, required for DON production in F. graminearum (Dyer et al. 2005). Furthermore, VDAG_09534, similar to the aflatoxin biosynthesis polyketide synthase gene, and several other members of the same secondary metabolism gene cluster exhibited strong induction in D-10-8F.

The results gathered have enabled us to propose a model on how activation and inactivation of $\mathrm{G}$ protein signaling is associated with the progression of the disease cycle in $V$. dahliae (Fig. 7). We propose that the upregulation of $\mathrm{G}$ proteinmediated signaling required for normal biotrophic interaction with the host takes place via repression of the RGS protein VdRGS1, a putative homolog of the developmental regulator FlbA. Hence, the transcription profile of mutant D-10-8F, in which VdRGS1 function is impaired, shows the induction of a number of activities that, in natural conditions, are associated with biotrophic growth on the host plant. These include production of secreted proteins required to penetrate plant tissues and counteract plant defense mechanisms, such as cell walldegrading enzymes, secreted proteases, and diverse elicitor proteins, and proteins involved in the metabolic adaptation to the limited nutrient availability encounter on plant tissues. Plant stress metabolites might, in fact, be used for nutrition by the fungus. Secondary metabolism associated with the production of toxins involved in the induction of wilt symptoms are also induced at this stage by activation of $G$ protein signaling through repression of VdRGS1 activity. According to our model, activation of VdRGS1 is required later during infection,

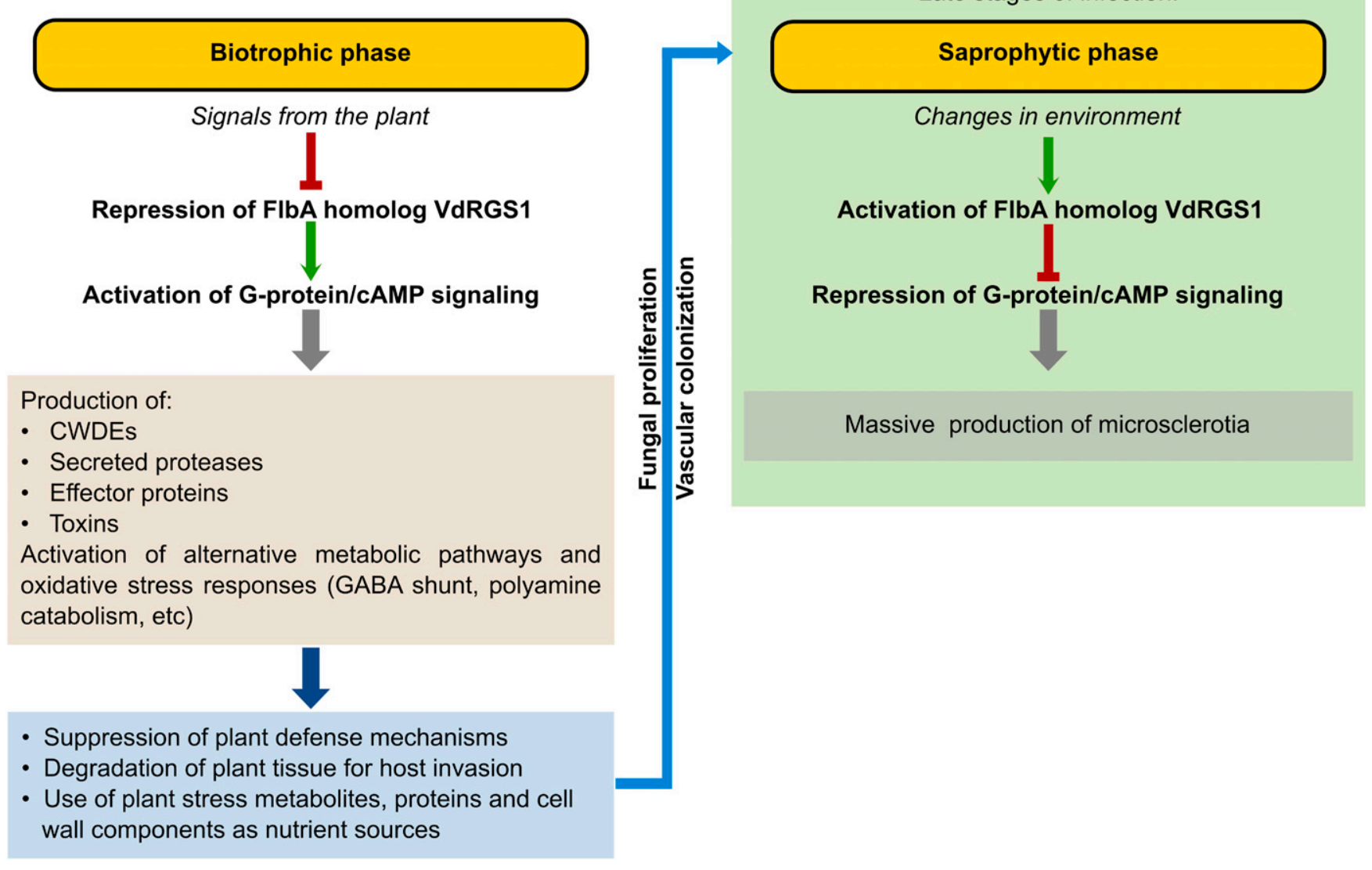

Fig. 7. Schematic representation of the role that activation or repression of G protein signaling plays in controlling the progression of the disease in Verticillium dahliae. VdRGS1 is a putative homolog of the negative regulator of G protein signaling FlbA, whose activity is required to block proliferation and induce development in Aspergillus sp. According to the proposed model, signals from the plant induce the inactivation of VdRGS1 at early stages of the biotrophic interaction. This, in turn, induces $\mathrm{G}$ protein signaling, which upregulates a number of activities that allow the fungus to overcome plant defenses, penetrate plant tissue, and acquire nutrients, thus proliferating and colonizing the vascular system. Pathogen proliferation and plant responses lead to environmental changes as the infection progresses, which are perceived by the fungal cells promoting the repression of G protein signaling through activation of VdRGS1. In this new context of downregulated $G$ protein signaling, fungal proliferation is blocked and the pathogen enters a phase of massive production of microsclerotia (MS). VdRGS1 is therefore proposed to play a critical role in controlling the transition between biotrophic growth and the saprophytic phase characterized by activation of the developmental pathway of MS formation. 
as the fungus enters into saprophytic growth and massive production of MS is induced. The association of an active $G$ protein-mediated signaling with proliferation in the host during biotrophic growth and of an active RGS protein, VdRGS1, with late infection in which large amounts of MS are produced is well supported by the data. Alteration of $G$ protein/cAMP signaling was previously shown to result in reduced virulence (Tzima et al. 2010, 2012). Here, we found that, in infections with $\Delta v d r g s 1$ strains, Verticillium wilt symptoms were clearly induced but, then, progression of the disease was halted. Furthermore, our model fits well with the observation that random mutant D-10-8F and $\Delta v d r g s 1$ strains are greatly impaired in MS production in in-vitro growth and is also in agreement with previous studies that showed that mutants with disrupted $G$ protein/cAMP signaling exhibited increased MS formation in culture (Tzima et al. 2010, 2012). The proposed model is also in agreement with a conserved role for FlbA-like proteins as negative regulators of fungal proliferation and positive regulators of developmental processes.

Further work will be required to attain a complete image of changes in VdRGS1 activity along the life cycle of $V$. dahliae. On cellophane membranes, $\Delta v d r g s l$ strains and the random mutant D-10-8F showed hyperpolarized growth, instead of producing the swollen hyphae resembling hyphopodia observed in the WT in this condition. Furthermore, VDAG_05860, a putative homolog of the homeobox TF gene $M o H O X 7$, required for the formation of appressoria in Magnaporthe oryzae (Kim et al. 2009), was down-regulated in mutant D-10-8F versus WT. These results suggest that VdRGS1 plays a role at initial stages of infection controlling the formation of hyphopodia, in addition to the above-discussed role as a master regulator of the switch between biotrophic growth and saprophytic development. Both roles are in agreement with a conserved function for VdRGS1 in repressing polar growth and fungal proliferation and inducing development. An in-depth analysis of the regulation of VdRGS1 activity will help in dissecting its role in the control of different developmental processes and clearly establish the involvement in virulence of these functions.

Genomic and transcriptomic approaches are providing a wealth of information that favors the generation of hypotheses regarding the regulation and cellular processes associated with development and virulence in phytopathogenic fungi. Functional characterization of individual genes to confirm their roles in the processes under study is critical to ultimately validate proposed hypotheses. Hence, efforts to simplify and speed up gene deletion methods in fungi are concomitant with the increasing availability of genomic resources. In that respect, for the deletion mutants produced in this work to functionally characterize specific genes, we have upgraded the previously reported method OSCAR (Paz et al. 2011). There are two key factors to develop methods in fungi suitable for highthroughput gene deletion approaches, i.e., first, to simplify the production of gene deletion constructs and, secondly, to favor the selection of deletion mutants upon fungal transformation. OSCAR was developed as a very simple method to produce gene deletion constructs compatible with ATMT that uses Gateway technology (Paz et al. 2011). In this work, we further improved OSCAR by including the negative selection marker $H S V t k$ to prevent ectopic integration of the T-DNA harboring the deletion construct. It has been reported that, when using this negative selection marker, transformants that are not deleted for the target gene might still be selected due to ectopic but incorrect integrations events of the T-DNA that result in inactivation of HSVtk and that the frequency of these incorrect integration events can vary (Khang et al. 2005). In this work, the performance of the modified version of OSCAR that incorporates HSVtk to combine rapid production of deletion constructs with negative selection of transformants in which the target gene has not been deleted was confirmed with the deletion of three genes. These include VdRGS1, the flbA homolog discussed above, and two genes identified as upregulated in D$10-8 \mathrm{~F}$ versus WT, vrgl and $v v s 1$, which encode a protein related to Ras-like GTPase and a HKK, respectively. In the whole, we analyzed 30 transformants by PCR and all but one showed deletion of the target gene. Furthermore, we analyzed six of the deletion mutants by Southern blot, showing that none of them harbored secondary random insertions of the T-DNA. These results confirm that introduction of HSVtk in the pOSCAR binary vector works efficiently in preventing ectopic integration of the deletion construct T-DNA upon fungal transformation. Unlike deletion mutants lacking VdRGSI, $\Delta v r g 1$ and $\Delta v v s 1$ strains did not exhibit phenotypic alterations. These results indicate that, although differentially expressed in WT versus D-10-8F, vvsl and vrgl do not play major roles in morphogenesis and virulence in $V$. dahliae. Alternatively, they might have redundant functions with other genes. The genomes of filamentous Ascomycota usually harbor multiple $\mathrm{HHK}$ genes, although the number varies in the different species (Hérivaux et al. 2016). No other HHKs have been functionally characterized in $V$. dahliae. OSCAR has been robust and efficient for production of deletion constructs for numerous genes in $V$. dahliae and other fungal species (Gold et al. 2017). The OSCAR update developed in this work can therefore be a gene deletion tool of broad interest for researchers working on fungal species.

\section{MATERIALS AND METHODS}

\section{Fungal strains and growth conditions.}

Verticillium dahliae strains used in this study include WT Dvd-T5 (Dobinson et al. 1996), random T-DNA insertion mutant D-10-8F, and deletion mutants lacking genes VdRGS1 (VDAG_00683),vrgl (VDAG_07039), and vvsl (VDAG_01858). Dvd-T5 was used as parental strain to generate both random and deletion mutants. Mutant strain D-10-8F is part of our collection of random T-DNA insertion mutants generated by collecting transformants that exhibit phenotypic alterations but are confirmed not to be deleted for the target gene upon ATMT of $V$. dahliae with OSCAR deletion constructs, as previously described (Sarmiento-Villamil et al. 2018). Strains were stored as conidial suspensions in $15 \%$ glycerol at $-80^{\circ} \mathrm{C}$ and were propagated on PDA (Difco). Mutant strains were grown on PDA supplemented with hygromycin B $(50 \mu \mathrm{g} / \mathrm{ml})$. Plates were incubated at $24^{\circ} \mathrm{C}$. Potato dextrose broth (Difco) or YEPS $(1 \%$ yeast extract, $2 \%$ bactopeptone, $2 \%$ sucrose) were used for liquid cultures to produce conidia for plant inoculations and fungal material for DNA extractions. To assay formation of MS in liquid culture, Czapek Dox liquid medium (modified) (Oxoid) was used. All liquid cultures were incubated at $24^{\circ} \mathrm{C}$ and $200 \mathrm{rpm}$.

\section{Generation of deletion mutants.}

Deletion constructs for $V d R G S 1, v r g 1$, and $v v s 1$ were generated using the OSCAR method but replacing the binary vector pOSCAR described by Paz et al. (2011) with a modified version termed pOSCAR-HSVtk. The latter vector contains the toxic gene $H S V t k$, used to prevent ectopic integration of the T-DNA upon transformation of the fungus. To construct pOSCAR-HSVtk, the HSVtk gene was amplified from plasmid pGKO2 (Khang et al. 2005) with primers pGKO2_F_656 and pGKO2_R_2576 (Supplementary Table S1), using Phusion High-Fidelity DNA polymerase (Thermo Fisher Scientific), and was cloned into pCR-Blunt (Thermo Fisher Scientific, 
Invitrogen). pGKO2 was a gift from $\mathrm{S}$. Kang (Addgene plasmid number 63617). HindIII recognition sites had been added to the 5' ends of both pGKO2_F_656 and pGKO2_R_2576 that allowed excising HSVtk from pCR-Blunt by digesting with HindIII. The HSVtk gene fragment was then cloned into the unique HindIII site of pOSCAR, located between the right border of the T-DNA and the BP clonase recombination sequence attP3 (Fig. 4A). To produce deletion constructs using the OSCAR method with this new vector, primer design, BP clonase reaction, and analysis of bacterial colonies to confirm deletion constructs were performed as previously described (Gold et al. 2017; Paz et al. 2011). Briefly, primers were designed to amplify $1 \mathrm{~kb}$ of the sequences flanking the ORF of the target genes. The two amplified fragments of each gene were mixed with pA-Hyg-GFP-OSCAR (Sarmiento-Villamil et al. 2018) and pOSCAR-HSVtk, and were incubated overnight in the presence of BP clonase. The BP clonase reaction was then used to transform One Shot OmniMAX 2 T1 Escherichia coli competent cells (Thermo Fisher Scientific, Invitrogen). Clones confirmed to contain the right deletion construct by PCR and restriction enzyme digestion analyses were selected. The deletion constructs were transformed into Agrobacterium tumefaciens AGL-1 using a standard heat-shock method (Weigel and Glazebrook 2006). Transformation of $V$. dahliae with deletion constructs was performed as described by Dobinson et al. (2004). In addition to hygromycin B $(50 \mu \mathrm{g} / \mathrm{ml})$, selection plates contained 5FU $(20 \mu \mathrm{g} / \mathrm{ml})$, which is converted to a toxic product by HSVtk (Khang et al. 2005; Wang et al. 2016).

Individual transformants were analyzed for gene replacement by PCR, using target gene-specific primers. The primer combinations used were FlbA_1808_F and FlbA_2358_R, Vrg1_368_F and Vrg1_978_R, and Vvs1_2306_F and Vvs1_2951_R, which amplified roughly 600-bp ORFs of VdRGS1, vrg1, and vvs1, respectively. All transformants were also analyzed with hygR primers Hyg_531_F and Hyg_975_R, as a positive control of amplification. Two deletion mutants were randomly selected for each gene and were further characterized by Southern blot analysis using digoxigenin (DIG)labeled probes derived from the target gene ORF and the hygR marker. Total DNA $(10 \mu \mathrm{g})$ from each sample to be tested was digested with the required restriction enzyme (HindIII, SphI, and BamHI for the analysis of $V d R G S 1$, vrgl, and $v v s 1$ deletion mutants, respectively), and was resolved on a $0.7 \%$ agarose gel. Digested DNAs were then transferred by capillarity to a positively charged nylon membrane (Roche). Labeled probes were generated by PCR, using the PCR DIG DNA labeling kit (Roche). To produce the gene-specific probes, the same primers used for the PCR analysis of transformants were used. To produce the hygR probe, primers Hyg-1 and Hyg-2 were used. Probes were detected with anti-DIG and chemiluminescent substrate CSPD (Roche). Each membrane was consecutively hybridized with the target gene-specific probe and the hygR probe. Probes derived from the gene ORFs detected no hybridization bands in the transformants, confirming gene deletion in all cases. The hygR probe detected a single hybridization band corresponding to the integration of the deletion construct by homologous recombination of the target gene flanks in all transformants tested, thus confirming absence of secondary integration events.

\section{Pathogenicity assays.}

Nicotiana benthamiana was used as a host for pathogenicity tests. Plants were inoculated by a root-dip method (Wellman 1939) with minor modifications. Tests were conducted using seedlings at the two- to three-true leaf stage and suspensions of $2.5 \times 10^{6}$ conidia per milliliter. For mock inoculations, plants were treated in the same way but with sterile water. Ten to 12 seedlings were used per treatment and pathogenicity assays were repeated at least three times. Disease severity was recorded according to a 0 to 3 scale: $0=$ healthy leaf, $1=$ chlorotic leaf, $2=$, leaf with wilting symptoms, and $3=$ fully necrotic leaf.

The disease severity (DS) for each strain in each replicate experiment was analyzed using the ranked data in PROC MIXED of SAS to obtain an ANOVA-F. As data were taken at four different times, 4, 5, 6, and 7 wpi, the DS was analyzed as a repeated measured experiment using a nonparametric statistical analysis. The ANOVA-F was calculated with an unstructured covariance model in which treatments, times, and the interaction treatment $\times$ time were fixed factors. The macros LD_CI and F1_LD_F1 were used to calculate the relative marginal effects for each treatment and $95 \%$ confidence intervals; two treatments were considered different when two CI did not overlap (Brunner et al. 2002; Shah and Madden 2004). An additional analysis to obtain orthogonal contrasts was also conducted between treatments at different times.

\section{Microarray analysis.}

For the microarray analysis, fungal total RNAs were extracted using Spectrum plant total RNA kit (Sigma-Aldrich), following manufacturer instructions. RNA integrity was confirmed by Tape Station using the R6K Screen Tape (Agilent Technologies). Verticillium dahliae custom arrays were constructed $(8 \times 15 \mathrm{~K}$ Gene Expression Microarray; ID 035922), each containing the 10,567 genes annotated in the publicly available sequence of strain VdLs.17. Labeled target sequences (cRNA) were produced using Two-Color Microarrays-Based Gene Expression Analysis v. 6.5 (Agilent Technologies). Dye swaps (Cy3 and Cy5) were performed for RNA amplified from each sample. Three biological replicates were performed for each sample. Verticillium dahliae custom array design, probe preparation, and hybridizations were performed by Bioarray (Elche). Chips were scanned using a DNA Microarray Scanner (Agilent Technologies) and were then processed with Feature Extraction v.10.7 software (Agilent Technologies), with the default variables. Outlier features on the arrays were flagged using the same software. Local background, normalization of signal intensities, and statistical analysis were conducted using the limma package, v. 3.16.1 of Bioconductor under R environment (Ritchie et al. 2015). Genes were assigned the same ID as in the annotated $V$. dahliae genome of strain VdLs. 17 for their identification. Genes were designated as differentially expressed when their differential expression ratios in the two strains were twofold or higher according to a parametric test, with $P$ value < 0.05 (moderated $t$ tests). They were classified into molecular function and biological process categories according to the GO Consortium database, using Blast2GO software (version 3.0) with hypergeometric test $P$ values $<0.001$. Subcellular localization and presence of signal peptides were predicted with DeepLoc-1.0 and SignalP-5.0, respectively.

\section{RT-qPCR.}

RT-qPCR was used to quantify expression of selected genes in WT and D-10-8F mutant strains. Fungal samples were prepared and total RNAs extracted as previously described for the microarray analysis. The mRNAs were transcribed to cDNA using MultiScribe Reverse transcription (Life Technologies). The qPCR reactions were carried out using $5 \times$ HOT FIREPol EvaGreen qPCR Mix Plus (Solis BioDyne), following manufacturer instructions, in a StepOne Thermal Cycler (Applied Biosystems) obtaining the cycle threshold (CT) values from the OneStep software. Relative expression levels were determined by the $\Delta \Delta \mathrm{CT}$ method based on two technical replicates and three biological replicates per sample and using actin and GADPH (glyceraldehyde 3-phosphate dehydrogenase) as housekeeping genes. 


\section{ACKNOWLEDGMENTS}

The authors thank G. V. Sandoya for his assistance with the statistical analysis of virulence data.

\section{AUTHOR-RECOMMENDED INTERNET RESOURCES}

DeepLoc-1.0 prediction tool: http://www.cbs.dtu.dk/services/DeepLoc Gene Ontology Consortium database: http://www.geneontology.org SignalP-5.0 server: http://www.cbs.dtu.dk/services/SignalP

\section{LITERATURE CITED}

Angelini, R., Cona, A., Federico, R., Fincato, P., Tavladoraki, P., and Tisi, A. 2010. Plant amine oxidases "on the move": An update. Plant Physiol. Biochem. 48:560-564.

Biswas, K., and Morschhäuser, J. 2005. The Mep2p ammonium permease controls nitrogen starvation-induced filamentous growth in Candida albicans. Mol. Microbiol. 56:649-669.

Bönnighausen, J., Gebhard, D., Kröger, C., Hadeler, B., Tumforde, T., Lieberei, R., Bergemann, J., Schäfer, W., and Bormann, J. 2015. Disruption of the GABA shunt affects mitochondrial respiration and virulence in the cereal pathogen Fusarium graminearum. Mol. Microbiol. 98:1115-1132.

Brunner, E., Domhof, S., and Langer, F. 2002. Nonparametric analysis of longitudinal data in factorial experiments. John Wiley and Sons, New York.

Dobinson, K. F., Grant, S. J., and Kang, S. 2004. Cloning and targeted disruption, via Agrobacterium tumefaciens-mediated transformation, of a trypsin protease gene from the vascular wilt fungus Verticillium dahliae. Curr. Genet. 45:104-110.

Dobinson, K. F., Tenuta, G. K., and Lazarovits, G. 1996. Occurrence of race 2 of Verticillium dahliae in processing tomato fields in southwestern Ontario. Can. J. Plant Pathol. 18:55-58.

Dumortier, F., Argüelles, J. C., and Thevelein, J. M. 1995. Constitutive glucose-induced activation of the Ras-cAMP pathway and aberrant stationary-phase entry on a glucose-containing medium in the Saccharomyces cerevisiae glucose-repression mutant hex2. Microbiology 141: 1559-1566.

Dyer, R. B., Plattner, R. D., Kendra, D. F., and Brown, D. W. 2005 Fusarium graminearum TRI14 is required for high virulence and DON production on wheat but not for DON synthesis in vitro. J. Agric. Food Chem. 53:9281-9287.

Gao, F., Zhou, B.-J., Li, G.-Y., Jia, P.-S., Li, H., Zhao, Y.-L., Zhao, P., Xia G. X., and Guo, H. S. 2010. A glutamic acid-rich protein identified in Verticillium dahliae from an insertional mutagenesis affects microsclerotial formation and pathogenicity. PLoS One 5:e15319.

Gasent-Ramírez, J. M., Codón, A. C., and Benítez, T. 1995. Characterization of genetically transformed Saccharomyces cerevisiae baker's yeasts able to metabolize melibiose. Appl. Environ. Microbiol. 61:2113-2121.

Gold, S. E., Paz, Z., García-Pedrajas, M. D., and Glenn, A. E. 2017. Rapid deletion production in fungi via agrobacterium-mediated transformation of OSCAR deletion constructs. J. Vis. Exp. 124:55239.

He, X. J., Li, X. L., and Li, Y. Z. 2015. Disruption of cerevisin via Agrobacterium tumefaciens-mediated transformation affects microsclerotia formation and virulence of Verticillium dahliae. Plant Pathol 64:1157-1167.

Hérivaux, A., So, Y. S., Gastebois, A., Latgé, J. P., Bouchara, J. P., Bahn, Y. S., and Papon, N. 2016. Major sensing proteins in pathogenic fungi: The hybrid histidine kinase family. PLoS Pathog. 12:e1005683.

Igarashi, K., and Kashiwagi, K. 2010. Characteristics of cellular polyamine transport in prokaryotes and eukaryotes. Plant Physiol. Biochem. 48: 506-512.

Khang, C. H., Park, S. Y., Lee, Y. H., and Kang, S. 2005. A dual selection based, targeted gene replacement tool for Magnaporthe grisea and Fusarium oxysporum. Fungal Genet. Biol. 42:483-492.

Kim, S., Park, S. Y., Kim, K. S., Rho, H. S., Chi, M. H., Choi, J., Park, J., Kong, S., Park, J., Goh, J., and Lee, Y. H. 2009. Homeobox transcription factors are required for conidiation and appressorium development in the rice blast fungus Magnaporthe oryzae. PLoS Genet. 5:e1000757.

Klimes, A., Dobinson, K. F., Thomma, B. P., and Klosterman, S. J. 2015. Genomics spurs rapid advances in our understanding of the biology of vascular wilt pathogens in the genus Verticillium. Annu. Rev. Phytopathol. 53:181-198.

Klosterman, S. J., Atallah, Z. K., Vallad, G. E., and Subbarao, K. V. 2009. Diversity, pathogenicity, and management of verticillium species. Annu. Rev. Phytopathol. 47:39-62.
Klosterman, S. J., Subbarao, K. V., Kang, S., Veronese, P., Gold, S. E., Thomma, B. P. H. J., Chen, Z., Henrissat, B., Lee, Y.-H., Park, J., GarcíaPedrajas, M. D., Barbara, D. J., Anchieta, A., de Jonge, R., Santhanam, P., Maruthachalam, K., Atallah, Z., Amyotte, S. G., Paz, Z., Inderbitzin, P., Hayes, R. J., Heiman, D. I., Young, S., Zeng, Q., Engels, R., Galagan, J., Cuomo, C. A., Dobinson, K. F., and Ma, L.-J. 2011. Comparative genomics yields insights into niche adaptation of plant vascular wilt pathogens. PLoS Pathog. 7:e1002137.

Krijgsheld, P., Nitsche, B. M., Post, H., Levin, A. M., Müller, W. H., Heck, A. J. R., Ram, A. F. J., Altelaar, A. F. M., and Wösten, H. A. B. 2013 Deletion of $f l b A$ results in increased secretome complexity and reduced secretion heterogeneity in colonies of Aspergillus niger. J. Proteome Res. 12:1808-1819.

Krüger, A., Vowinckel, J., Mülleder, M., Grote, P., Capuano, F., Bluemlein, K., and Ralser, M. 2013. Tpo1-mediated spermine and spermidine export controls cell cycle delay and times antioxidant protein expression during the oxidative stress response. EMBO Rep. 14:1113-1119.

Kulkarni, R. D., Thon, M. R., Pan, H., and Dean, R. A. 2005. Novel Gprotein-coupled receptor-like proteins in the plant pathogenic fungus Magnaporthe grisea. Genome Biol. 6:R24.

Lee, J. D., and Kolattukudy, P. E. 1995. Molecular cloning of the cDNA and gene for an elastinolytic aspartic proteinase from Aspergillus fumigatus and evidence of its secretion by the fungus during invasion of the host lung. Infect. Immun. 63:3796-3803.

Li, L., Wright, S. J., Krystofova, S., Park, G., and Borkovich, K. A. 2007. Heterotrimeric $\mathrm{G}$ protein signaling in filamentous fungi. Annu. Rev. Microbiol. 61:423-452.

Lorenz, M. C., and Heitman, J. 1998. The MEP2 ammonium permease regulates pseudohyphal differentiation in Saccharomyces cerevisiae. EMBO J. 17:1236-1247.

Luo, X., Mao, H., Wei, Y., Cai, J., Xie, C., Sui, A., Yang, X., and Dong, J. 2016. The fungal-specific transcription factor Vdpf influences conidia production, melanized microsclerotia formation and pathogenicity in Verticillium dahliae. Mol. Plant Pathol. 17:1364-1381.

Maruthachalam, K., Klosterman, S. J., Kang, S., Hayes, R. J., and Subbarao, K. V. 2011. Identification of pathogenicity-related genes in the vascular wilt fungus Verticillium dahliae by Agrobacterium tumefaciens-mediated T-DNA insertional mutagenesis. Mol. Biotechnol. 49:209-221

Mo, H., Wang, X., Zhang, Y., Zhang, G., Zhang, J., and Ma, Z. 2015. Cotton polyamine oxidase is required for spermine and camalexin signalling in the defence response to Verticillium dahliae. Plant J. 83:962-975.

Morschhäuser, J. 2011. Nitrogen regulation of morphogenesis and protease secretion in Candida albicans. Int. J. Med. Microbiol. 301:390-394.

Mullins, E. D., and Kang, S. 2001. Transformation: A tool for studying fungal pathogens of plants. Cell. Mol. Life Sci. 58:2043-2052.

Naglik, J., Albrecht, A., Bader, O., and Hube, B. 2004. Candida albicans proteinases and host/pathogen interactions. Cell. Microbiol. 6:915-926.

Paul, J. A., Wallen, R. M., Zhao, C., Shi, T., and Perlin, M. H. 2018 Coordinate regulation of Ustilago maydis ammonium transporters and genes involved in mating and pathogenicity. Fungal Biol. 122:639-650.

Paz, Z., García-Pedrajas, M. D., Andrews, D. L., Klosterman, S. J., BaezaMontañez, L., and Gold, S. E. 2011. One step construction of Agrobacterium-recombination-ready-plasmids (OSCAR), an efficient and robust tool for ATMT based gene deletion construction in fungi. Fungal Genet. Biol. 48:677-684.

Reignault, P., Valette-Collet, O., and Boccara, M. 2008. The importance of fungal pectinolytic enzymes in plant invasion, host adaptability and symptom type. Eur. J. Plant Pathol. 120:1-11.

Ritchie, M. E., Phipson, B., Wu, D., Hu, Y., Law, C. W., Shi, W., and Smyth, G. K. 2015. limma powers differential expression analyses for RNAsequencing and microarray studies. Nucleic Acids Res. 43:e47.

Rutherford, J. C., Bahn, Y. S., van den Berg, B., Heitman, J., and Xue, C. 2019. Nutrient and stress sensing in pathogenic yeasts. Front Microbiol. $8: 442$.

Santhanam, P. 2012. Random insertional mutagenesis in fungal genomes to identify virulence factors. Methods Mol. Biol. 835:509-517.

Santhanam, P., Boshoven, J. C., Salas, O., Bowler, K., Islam, M. T., Saber, M. K., van den Berg, G. C., Bar-Peled, M., and Thomma, B. P. 2017 Rhamnose synthase activity is required for pathogenicity of the vascular wilt fungus Verticillium dahliae. Mol. Plant Pathol. 18:347-362.

Santhanam, P., van Esse, H. P., Albert, I., Faino, L., Nürnberger, T., and Thomma, B. P. 2013. Evidence for functional diversification within a fungal NEP1-like protein family. Mol. Plant-Microbe Interact. 26:278-286.

Sarmiento-Villamil, J. L., Prieto, P., Klosterman, S. J., and García-Pedrajas, M. D. 2018. Characterization of two homeodomain transcription factors with critical but distinct roles in virulence in the vascular pathogen Verticillium dahliae. Mol. Plant Pathol. 19:986-1004. 
Shah, D. A., and Madden, L. V. 2004. Nonparametric analysis of ordinal data in designed factorial experiments. Phytopathology 94:33-43.

Siegel, D., Gustafson, D. L., Dehn, D. L., Han, J. Y., Boonchoong, P., Berliner, L. J., and Ross, D. 2004. NAD(P)H:quinone oxidoreductase 1: Role as a superoxide scavenger. Mol. Pharmacol. 65:1238-1247.

Solomon, P. S., and Oliver, R. P. 2002. Evidence that gamma-aminobutyric acid is a major nitrogen source during Cladosporium fulvum infection of tomato. Planta 214:414-420.

Soloviev, D. A., Jawhara, S., and Fonzi, W. A. 2011. Regulation of innate immune response to Candida albicans infections by $\alpha \mathrm{M} \beta 2$-Pra $1 \mathrm{p}$ interaction. Infect. Immun. 79:1546-1558.

Turrión-Gómez, J. L., Eslava, A. P., and Benito, E. P. 2010. The flavohemoglobin BCFHG1 is the main NO detoxification system and confers protection against nitrosative conditions but is not a virulence factor in the fungal necrotroph Botrytis cinerea. Fungal Genet. Biol. 47: 484-496.

Tzima, A., Paplomatas, E. J., Rauyaree, P., and Kang, S. 2010. Roles of the catalytic subunit of cAMP-dependent protein kinase A in virulence and development of the soilborne plant pathogen Verticillium dahliae. Fungal Genet. Biol. 47:406-415.

Tzima, A. K., Paplomatas, E. J., Tsitsigiannis, D. I., and Kang, S. 2012. The $\mathrm{G}$ protein $\beta$ subunit controls virulence and multiple growth- and development-related traits in Verticillium dahliae. Fungal Genet. Biol. 49:271-283.

Uhl, M. A., and Miller, J. F. 1996. Central role of the BvgS receiver as a phosphorylated intermediate in a complex two-component phosphorelay. J. Biol. Chem. 271:33176-33180.

Valdés-Santiago, L., Cervantes-Chávez, J. A., León-Ramírez, C. G., and Ruiz-Herrera, J. 2012. Polyamine metabolism in fungi with emphasis on phytopathogenic species. J. Amino Acids 2012:837932.

Valdés-Santiago, L., and Ruiz-Herrera, J. 2014. Stress and polyamine metabolism in fungi. Front Chem. 1:42.

Vallad, G. E., and Subbarao, K. V. 2008. Colonization of resistant and susceptible lettuce cultivars by a green fluorescent protein-tagged isolate of Verticillium dahliae. Phytopathology 98:871-885

Wang, S., Xing, H., Hua, C., Guo, H. S., and Zhang, J. 2016. An improved single-step cloning strategy simplifies the Agrobacterium tumefaciensmediated transformation (ATMT)-based gene-disruption method for Verticillium dahliae. Phytopathology 106:645-652.

Weigel, D., and Glazebrook, J. 2006. Transformation of Agrobacterium using the freeze-thaw method. Cold Spring Harb. Protoc. 2006: pdb.prot4666.

Wellman, F. L. 1939. A technique for studying host resistance and pathogenicity in tomato Fusarium wilt. Phytopathology 29:945-956.

Xie, C., Li, Q., and Yang, X. 2017. Characterization of VdASP F2 secretory factor from Verticillium dahliae by a fast and easy gene knockout system. Mol. Plant-Microbe Interact. 30:444-454.
Xu, J., Wang, X., Li, Y., Zeng, J., Wang, G., Deng, C., and Guo, W. 2018. Host-induced gene silencing of a regulator of $\mathrm{G}$ protein signalling gene (VdRGS1) confers resistance to Verticillium wilt in cotton. Plant Biotechnol. J. 16:1629-1643.

Yadeta, K. A., and Thomma, B. P. H. J. 2013. The xylem as battleground for plant hosts and vascular wilt pathogens. Front. Plant Sci. 4:97.

Yang, Y., Zhang, Y., Li, B., Yang, X., Dong, Y., and Qiu, D. 2018. A Verticillium dahliae pectate lyase induces plant immune responses and contributes to virulence. Front. Plant Sci. 9:1271.

Ye, Y., Minami, A., Igarashi, Y., Izumikawa, M., Umemura, M., Nagano, N., Machida, M., Kawahara, T., Shin-Ya, K., Gomi, K., and Oikawa, H 2016. Unveiling the biosynthetic pathway of the ribosomally synthesized and post-translationally modified peptide ustiloxin B in filamentous fungi. Angew. Chem. Int. Ed. Engl. 55:8072-8075.

Yu, J. H., Wieser, J., and Adams, T. H. 1996. The Aspergillus FlbA RGS domain protein antagonizes $\mathrm{G}$ protein signaling to block proliferation and allow development. EMBO J. 15:5184-5190.

Zhang, J., Zhang, Y., Yang, J., Kang, L., EloRM, A. M., Zhou, H., and Zhao, J. 2019. The $\alpha-1,6$-mannosyltransferase $\mathrm{VdOCH} 1$ plays a major role in microsclerotium formation and virulence in the soil-borne pathogen Verticillium dahliae. Fungal Biol. 123:539-546.

Zhang, W. Q., Gui, Y. J., Short, D. P. G., Li, T. G., Zhang, D. D., Zhou, L., Liu, C., Bao, Y. M., Subbarao, K. V., Chen, J. Y., and Dai, X. F. 2018. Verticillium dahliae transcription factor VdFTF1 regulates the expression of multiple secreted virulence factors and is required for full virulence in cotton. Mol. Plant Pathol. 19:841-857.

Zhang, Y., Gao, Y., Liang, Y., Dong, Y., Yang, X., Yuan, J., and Qiu, D. 2017. The Verticillium dahliae SnodProt1-like protein VdCP1 contributes to virulence and triggers the plant immune system. Front. Plant Sci. 8:1880.

Zhang, Y. L., Li, Z. F., Feng, Z. L., Feng, H. J., Zhao, L. H., Shi, Y. Q., Hu, X. P., and Zhu, H. Q. 2015a. Isolation and functional analysis of the pathogenicity-related gene VdPR3 from Verticillium dahliae on cotton. Curr. Genet. 61:555-566

Zhang, Z., Wang, J., Chai, R., Qiu, H., Jiang, H., Mao, X., Wang, Y., Liu, F. and Sun, G. 2015b. An S-(hydroxymethyl)glutathione dehydrogenase is involved in conidiation and full virulence in the rice blast fungus Magnaporthe oryzae. PLoS One 10:e0120627.

Zheng, H., Zhou, L., Dou, T., Han, X., Cai, Y., Zhan, X., Tang, C., Huang, J., and $\mathrm{Wu}, \mathrm{Q}$. 2010. Genome-wide prediction of $\mathrm{G}$ protein-coupled receptors in Verticillium spp. Fungal Biol. 114:359-368.

Zhou, B.-J., Jia, P.-S., Gao, F., and Guo, H.-S. 2012. Molecular characterization and functional analysis of a necrosis- and ethyleneinducing, protein-encoding gene family from Verticillium dahliae. Mol. Plant-Microbe Interact. 25:964-975.

Zhou, T. T., Zhao, Y. L., and Guo, H. S. 2017. Secretory proteins are delivered to the septin-organized penetration interface during root infection by Verticillium dahliae. PLoS Pathog. 13:e1006275. 